\title{
Study of energy response and resolution of the ATLAS Tile Calorimeter to hadrons of energies from 16 to $30 \mathrm{GeV}$
}

Jalal Abdallah ${ }^{1}$, Stylianos Angelidakis ${ }^{2}$, Giorgi Arabidze ${ }^{3}$, Nikolay Atanov ${ }^{4}$, Johannes Bernhard $^{5}$, Roméo Bonnefoy $^{6}$, Jonathan Bossio ${ }^{7}$, Ryan Bouabid ${ }^{8}$, Fernando Carrio ${ }^{9}$, Tomas Davidek ${ }^{10}$, Michal Dubovsky ${ }^{11}$, Luca Fiorini $^{9}$, Francisco Brandan Garcia Aparisi ${ }^{9}$, Tancredi Carli ${ }^{5}$, Alexander Gerbershagen ${ }^{5}$, Hazal Goksu ${ }^{8}$, Haleh Hadavand ${ }^{1}$, Siarhei Harkusha ${ }^{12}$, Dingane Hlaluku ${ }^{13}$, Michael James Hibbard ${ }^{1}$, Kevin Hildebrand ${ }^{8}$, Aliaksei Hrynevich ${ }^{14}$, Juansher Jejelava ${ }^{15}$, Andrey Kamenshchikov ${ }^{16}$, Stergios Kazakos ${ }^{17}$, Tomas Kello ${ }^{10}$, Ilya Korolkov ${ }^{17}$, Yuri Kulchitsky ${ }^{12}$, Hadar Lazar ${ }^{8}$, Nthabiseng Lekalakala ${ }^{13}$, Jared Little ${ }^{1}$, Romain Madar ${ }^{6}$, Samuel Manen ${ }^{6}$, Filipe Martins ${ }^{18}$, Thabo Masuku ${ }^{13}$, Irakli Minashvili ${ }^{4}$, Tigran Mkrtchyan ${ }^{19, a}$, Michaela Mlynarikova $^{20}$, Seyedali Moayedi ${ }^{1}$, Stanislav Nemecek ${ }^{21}$, Lawrence Nodulman ${ }^{22}$, Robert Oganezov ${ }^{23}$, Mats Joakim Robert Olsson ${ }^{24}$, Mark Oreglia ${ }^{8}$, Priscilla Pani ${ }^{25}$, Alexander Paramonov ${ }^{22}$, Ruth Pottgen ${ }^{26}$, Tres Reid ${ }^{8}$, Sergi Rodriguez Bosca ${ }^{9}$, Andrea Rodriguez Perez ${ }^{17}$, Rachel Christine Rosten ${ }^{27}$, Puja Saha ${ }^{20}$, Claudio Santoni $^{6, b}$, Laura Sargsyan ${ }^{23}$, Douglas Michael Schaefer ${ }^{8}$, Nikolay Shalanda ${ }^{16}$, Andrew Caldon Smith ${ }^{28}$, Alexander Solodkov ${ }^{16}$, Oleg Solovyanov ${ }^{16}$, Pavel Starovoitov ${ }^{19}$ (D), Evgeny Starchenko ${ }^{16}$, Petr Tas ${ }^{10}$, Viacheslav Tereshchenko ${ }^{4}$, Sijiye Humphry Tlou ${ }^{13}$, Michael Ughetto ${ }^{29}$, Lea Uhliarova ${ }^{10}$, Giulio Usai ${ }^{1}$, Eduardo Valdes Santurio ${ }^{29}$, Alberto Valero Biot ${ }^{9}$, Guido Volpi $^{17}$, Tamar Zakareishvili ${ }^{30}$, Pedro Diego Zuccarello ${ }^{9}$

${ }^{1}$ Department of Physics, University of Texas at Arlington, Arlington, TX, USA

2 Physics Department, National and Kapodistrian University of Athens, Athens, Greece

${ }^{3}$ Department of Physics and Astronomy, Michigan State University, East Lansing, MI, USA

${ }^{4}$ Joint Institute for Nuclear Research, Dubna, Russia

${ }^{5}$ CERN, Geneva, Switzerland

${ }^{6}$ LPC, Université Clermont Auvergne, CNRS/IN2P3, Clermont-Ferrand, France

${ }^{7}$ Department of Physics, McGill University, Montreal, QC, Canada

${ }^{8}$ Enrico Fermi Institute, University of Chicago, Chicago, IL, USA

${ }^{9}$ Instituto de Física Corpuscular (IFIC), Centro Mixto Universidad de Valencia, CSIC, Valencia, Spain

${ }^{10}$ Faculty of Mathematics and Physics, Charles University, Prague, Czech Republic

${ }^{11}$ Faculty of Mathematics, Physics and Informatics, Comenius University, Bratislava, Slovakia

${ }^{12}$ B.I. Stepanov Institute of Physics, National Academy of Sciences of Belarus, Minsk, Belarus

13 School of Physics, University of the Witwatersrand, Johannesburg, South Africa

${ }^{14}$ Research Institute for Nuclear Problems of Byelorussian State University, Minsk, Belarus

${ }^{15}$ E. Andronikashvili Institute of Physics, Iv. Javakhishvili Tbilisi State University, Tbilisi, Georgia

${ }^{16}$ Institute for High Energy Physics of the National Research Centre Kurchatov Institute, Protvino, Russia

${ }^{17}$ Institut de Física d'Altes Energies (IFAE), Barcelona Institute of Science and Technology, Barcelona, Spain

${ }^{18}$ Laboratório de Instrumentação e Física Experimental de Partículas - LIP, Lisbon, Portugal

${ }^{19}$ Kirchhoff-Institut für Physik, Ruprecht-Karls-Universität Heidelberg, Heidelberg, Germany

${ }^{20}$ Department of Physics, Northern Illinois University, DeKalb, IL, USA

${ }^{21}$ Institute of Physics of the Czech Academy of Sciences, Prague, Czech Republic

${ }^{22}$ High Energy Physics Division, Argonne National Laboratory, Argonne, IL, USA

${ }^{23}$ Alikhanyan National Science Laboratory, Yerevan, Armenia

${ }^{24}$ Department of Physics and Astronomy, University of California Irvine, Irvine, CA, USA

${ }^{25}$ Deutsches Elektronen-Synchrotron DESY, Hamburg and Zeuthen, Germany

${ }^{26}$ Fysiska institutionen, Lunds universitet, Lund, Sweden

27 Ohio State University, Columbus, OH, USA

${ }^{28}$ Nevis Laboratory, Columbia University, Irvington, NY, USA

${ }^{29}$ Oskar Klein Centre, Stockholm, Sweden

${ }^{30}$ High Energy Physics Institute, Tbilisi State University, Tbilisi, Georgia 
Abstract Three spare modules of the ATLAS Tile Calorimeter were exposed to test beams from the Super Proton Synchrotron accelerator at CERN in 2017. The detector's measurements of the energy response and resolution to positive pions and kaons, and protons with energies ranging from 16 to $30 \mathrm{GeV}$ are reported. The results have uncertainties of a few percent. They were compared to the predictions of the Geant4-based simulation program used in ATLAS to estimate the response of the detector to proton-proton events at the Large Hadron Collider. The determinations obtained using experimental and simulated data agree within the uncertainties.

\section{Introduction}

Three spare modules of the Tile Calorimeter (TileCal) of the ATLAS experiment [1], two long-barrels and one extendedbarrel, were exposed to muon, electron pion, kaon and proton beams with different energies and incident angles at test beams (TBs) in 2017 [2].

The role of the hadron calorimeter in ATLAS is to measure the energy and the angle of isolated hadrons and jets. To achieve good performance, the study of the sub-detector response to isolated hadrons is important. In this paper, the measurements of the calorimeter response and resolution to positive pion and kaon, and proton beams with energies ranging from 16 to $30 \mathrm{GeV}$ are presented. The results are compared with the ones obtained analyzing simulated data produced using the ATLAS Geant4 toolkit [3], [4] and [5]. The experimental setup including the beam line counters and the detector is described in Sect. 2. The data sets, event selections and reconstruction of the particle energies in the case of experimental and simulated data are presented in Sects. 3 and 4 , respectively. The determinations of the calorimeter responses and resolutions are discussed in Sect. 5. The results are compared with hadronic cascade model predictions in Sect. 6. The conclusions are stated in Sect. 7.

\section{The experimental setup}

\subsection{The beam line}

The measurements discussed in this paper were performed using tertiary particle beams at the $\mathrm{H} 8$ line in the North Area of CERN [2]. Secondary beams are produced by hitting $400 \mathrm{GeV}$ protons from the Super Proton Synchrotron (SPS) accelerator on a $100 \mathrm{~mm}$ thick T4 target made of beryllium (primary target). Tertiary beams can be produced by using

\footnotetext{
a e-mail: tigran.mkrtchyan@cern.ch (corresponding author)

be-mail: claudio.santoni@cern.ch
}

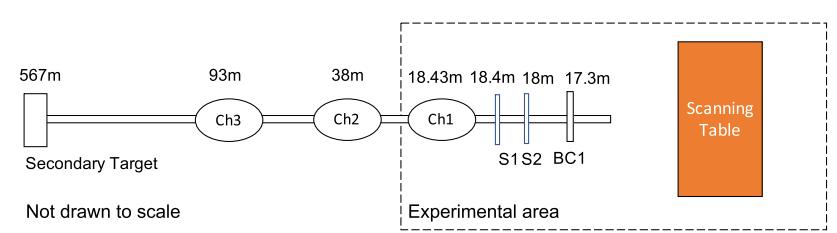

Fig. 1 Schematic layout of the H8 beam line detectors. The distances of the beam line components and of the secondary target from the scanning Table (on which was placed the TB calorimeter) setup are shown

Table 1 Cherenkov radiator materials and gas pressure values set for the different beam energies

\begin{tabular}{lcll}
\hline Cherenkov Counter & $\mathrm{Ch} 1$ & $\mathrm{Ch} 2$ & $\mathrm{Ch} 3$ \\
\hline Radiator Material & $\mathrm{CO}_{2}$ & $\mathrm{CO}_{2}$ & $\mathrm{He}$ \\
\hline$E_{\text {beam }}(\mathrm{GeV})$ & Pressure (bar) & & \\
\hline 16 & 0.19 & 0.75 & 2.6 \\
18 & 0.19 & 0.75 & 2.6 \\
20 & 0.19 & 0.75 & 2.6 \\
30 & 0.3 & 2.0 & 2.6 \\
\hline
\end{tabular}

secondary targets located at about $130 \mathrm{~m}$ downstream from the T4 target. A large spectrometer constructed of four Main Bend North Area dipole magnets is used to define the particle momenta. Beam particles can have energies from 10 to $350 \mathrm{GeV}$. Beam intensity decreases dramatically at the low energies. Depending on the desired properties of the tertiary beams, different secondary targets are used. For mixed hadron enriched tertiary beams, the secondary target is made of $300 \mathrm{~mm}$ thick copper. Additionally, a $6 \mathrm{~mm}$ thick lead absorber located $270 \mathrm{~m}$ downstream of the target absorbs the electrons while allowing most of the hadrons to pass through. For electron enriched tertiary beams, the secondary target is made of $400 \mathrm{~mm}$ thick aluminum and the lead absorber is placed immediately behind it.

The layout of the beam line detectors is shown in Fig. 1. The transverse beam profile was monitored by the wire chamber BC1 [6]. Two scintillating counters, S1 and S2, each with an active surface of $5 \times 5 \mathrm{~cm}^{2}$ [7], were used in coincidence to trigger the data acquisition (Physics Trigger) and to provide the trigger timing. These two detectors were also used to reject beam particles interacting upstream of the detector. The Cherenkov counters Ch1, Ch2 and Ch3 allowed identification of beam particles. The counters $\mathrm{Ch} 1$ and $\mathrm{Ch} 3$ distinguish electrons and pions from kaons and protons, while $\mathrm{Ch} 2$ distinguishes between kaons and protons. The counters $\mathrm{Ch} 1$ and $\mathrm{Ch} 2$ were filled with $\mathrm{CO}_{2}$ while $\mathrm{Ch} 3$ was filled with He. The higher pressure within $\mathrm{Ch} 2$ allows it to be used to separate kaons and protons. More details can be found in Ref. [7]. The pressure values of $\mathrm{Ch} 1, \mathrm{Ch} 2$ and $\mathrm{Ch} 3$ set for the different beam energies are reported in Table 1. 


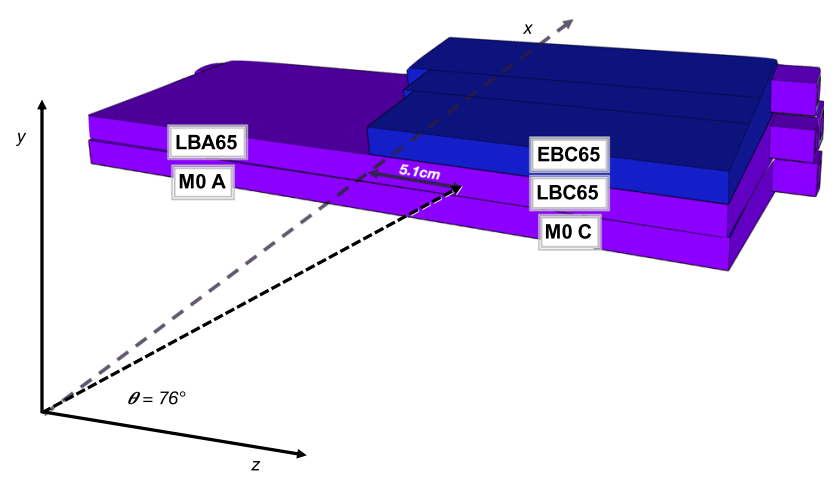

Fig. 2 Schematic view of the TileCal modules as stacked on the scanning table at the $\mathrm{H} 8$ beam line. The names of the super-drawers and the direction and the interaction point of the particle beams in the detector are shown

\subsection{The detector}

The TB setup, shown in Fig. 2, consists of three spare ATLAS modules [1] of TileCal - two long-barrels and one extended-barrel. These modules are stacked on a scanning table (Fig. 1) that is capable of placing them at different positions and angles with respect to the incoming beam particles. An extended-barrel consists of one super-drawer while a long-barrel consists of two super-drawers. In the figure they are named M0 A and M0 C (module at the bottom), LBA65 and LBC65 (module in the middle) and EBC65 (module at the top). The super-drawers LBA65, LBC65 and M0 A were equipped with different upgraded front-end electronics systems proposed for the ATLAS Large Hadron Collider (LHC) Phase-II operations [8]. The super-drawers EBC65 and M0 $\mathrm{C}$ were equipped with the electronics installed currently in ATLAS [1].

As shown in Fig. 3, the modules have a periodic structure of steel plates and scintillating tiles perpendicular to the $z$ axis. Wavelength-shifting fibres transmit light produced in the tiles to the PhotoMultipliers (PMTs) [9]. Each module has a three-dimensional cell structure defined by grouping optical fibres connected to the same PMT [10]. In general, two PMTs read-out a cell and the signals are summed up to provide the cell response. A structure of three cell layers parallel to the $z$ axis is obtained. The cell layers $\mathrm{A}, \mathrm{BC}$ and $\mathrm{D}$ in half long-barrel and A, B and D in extended barrel are shown in Fig. 4.

As in the ATLAS detector at the LHC, the energy deposited in a cell of the TB detector, $E_{\mathrm{c}}^{\text {raw }}$, was determined making use of the Optimal Fit method [11]. The linearity of the ADC's is determined using the Charge Injection System (CIS) [12]. The inter-calibration of the different calorimeter cells was obtained by equalizing the PMT current induced by movable radioactive ${ }^{137} \mathrm{Cs}$ sources that cross every row of scintillating tiles near the edges (Fig. 3). Since the scintillating tile response depends on the impact point position

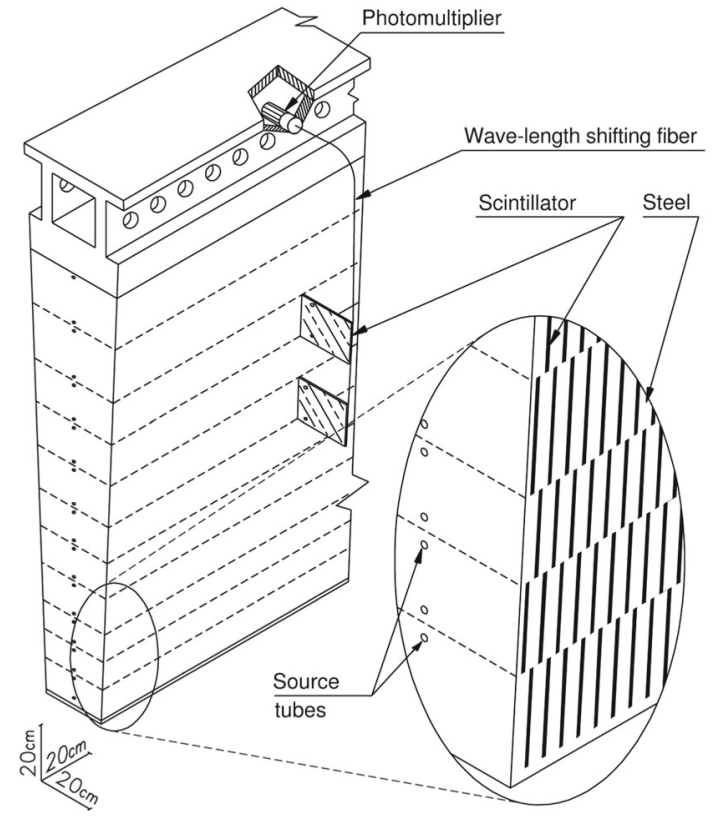

Fig. 3 Mechanical structure of a TileCal module, showing the slots in the steel for scintillating tiles and the method of light collection by wavelength-shifting fibres to PMTs. The holes for radioactive source tubes that traverse the module perpendicularly to the iron plates and scintillating tiles are also shown

of the particle in the tile and on the tile size, correction factors were applied for each layer of the calorimeter. Those values were determined from prior TB data, which measured the response to muons impinging on the calorimeter with a direction parallel to the $z$ axis (Fig. 2), and from the measurements obtained using a $\mathrm{Sr}$ source [12]. The scale of the reconstructed cell energy, $C_{\mathrm{c}}^{\mathrm{EM}}=1.05 \mathrm{pC} / \mathrm{GeV}$, was obtained using electron beams incident at the centre of each cell with an angle of $20^{\circ}$ with respect to the cell surface normal. The estimated uncertainty is $\Delta C_{\mathrm{c}}^{\mathrm{EM}}=2.4 \%$ [12]. The analysis of the muon and electron TB data collected in the 2017 TB [8] produced performance results that agree with the ones obtained using previous TBs [12] and with in-situ measurements in ATLAS [13].

To be consistent, the Optimal Fit method [11] was applied also to reconstruct the energy deposited in the cells in the case of simulated events. The scale of the cell energy measurements was obtained using the response to simulated electrons.

The energy deposited by the beam particles incident the detector, $E^{\text {raw }}$, was determined as the sum of the energy measured in the calorimeter cells. 


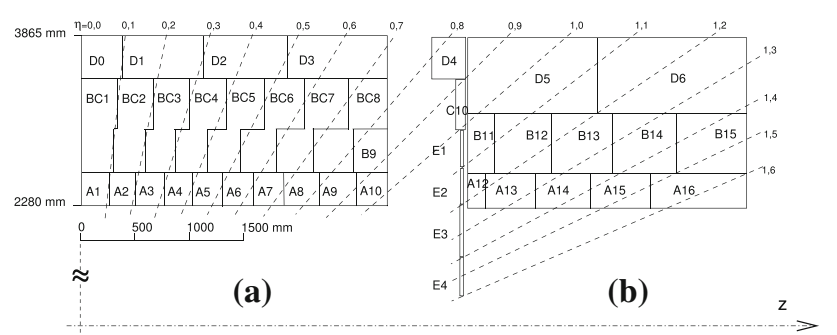

Fig. 4 The cell structure in the right half the long barrel (a) and extended-barrel (b) module of the calorimeter. The left half of the long barrel is not shown as the long barrel is symmetrical. Solid lines show the cell boundaries formed by grouping optical fibers from the tiles for read out by separate PMTs. The dashed lines represent fixed pseudorapidity values

\section{Analysis of experimental data}

The results discussed in this paper were obtained exposing the TB calorimeter setup to enriched tertiary positive hadron beams with energy, $E_{\text {beam }}$, equal to $16,18,20$ and $30 \mathrm{GeV}$. As shown in Fig. 2, the beams hit at the centre of the cell A3 of the super-drawer LBC65 with an azimuth angle $\phi=0$ and polar angle $\theta$ of about $76^{\circ}$, corresponding to a pseudorapidity value $\eta=0.25$ [1]. (Fig. 4). The angle from the calorimeter module normal is equal to $14^{\circ}$. The numbers of events collected during the data taking period are reported in Table 2 (Physics Trigger).

\subsection{Collimated single-particle events}

Collimated single-particle events were first selected using beam detectors upstream of the TB calorimeter setup. The selection criteria on the beam line scintillating counters signals, $E_{\mathrm{S} 1}$ and $E_{\mathrm{S} 2}$, were established using of the responses of $\mathrm{S} 1$ and $\mathrm{S} 2$ to muons. Muon events were recognized by requiring the energy deposited in the module LBC65 to be compatible with the one deposited by a minimum ionizing particle. The retained events satisfy the criteria:

$E_{\mathrm{S} 1}<2 \times E_{\mathrm{S} 1}^{\text {m.p. }}(\mu)$

and

$E_{\mathrm{S} 2}<2 \times E_{\mathrm{S} 2}^{\mathrm{m} \cdot \mathrm{p} .}(\mu)$

where the quantities $E_{\mathrm{S} 1}^{\text {m.p. }}(\mu)$ and $E_{\mathrm{S} 2}^{\text {m.p. }}(\mu)$ are the most probable values of the $\mathrm{S} 1$ and $\mathrm{S} 2$ muon signal distributions, respectively. The selection criteria, especially useful for electron studies, remove particles that initiated a shower upstream of the calorimeter, as well as multi-particle beam events. The number of events retained after the application of the criterion are reported in Table 2 (Selection 1).
Table 2 Numbers of experimental data events collected (Physics Trigger) and retained in the analysis selection steps for each of the four beam energies. The number of events identified as electrons, pions, kaons and protons is reported. Selection criteria and determination of statistical uncertainties on the number of electrons and pions are discussed in the text

\begin{tabular}{lll}
\hline$E_{\text {beam }}(\mathrm{GeV})$ & 16 & 18 \\
\hline Physics trigger & 694658 & 944460 \\
Selection 1 & 656262 & 895863 \\
Selection 2 & 552179 & 771513 \\
Selection 3 & 501013 & 700590 \\
$e / \pi$ & 385718 & 556782 \\
$K / p$ & 86635 & 133071 \\
Electrons & $67647 \pm 9198$ & $70834 \pm 3665$ \\
Pions & $318071 \mp 9198$ & $485948 \mp 3665$ \\
Kaons & 2372 & 4674 \\
Protons & 84263 & 128397 \\
\hline$E_{\text {beam }}(\mathrm{GeV})$ & 20 & 30 \\
\hline Physics trigger & 1226756 & 1297099 \\
Selection 1 & 1155580 & 1230470 \\
Selection 2 & 935131 & 1069709 \\
Selection 3 & 777386 & 983892 \\
$e / \pi$ & 611687 & 723286 \\
$K / p$ & 154181 & 137119 \\
Electrons & $62137 \pm 3548$ & $28288 \pm 2481$ \\
Pions & $549550 \mp 3548$ & $694998 \mp 2481$ \\
Kaons & 6782 & 11296 \\
Protons & 147399 & 125823 \\
\hline & & \\
\hline & & \\
\hline & &
\end{tabular}

The beam chamber $\mathrm{BC} 1$ allows a determination of the transverse beam impact point coordinates, $x_{\mathrm{BC} 1}$ and $y_{\mathrm{BC} 1}$. Events with a beam trajectory far away from the beam axis were rejected because the beam particles might have scattered upstream and therefore be off-energy. Gaussian functions were fitted to the distributions of each data set to determine the peak values $x_{\mathrm{BC} 1}^{\text {peak }}$ and $y_{\mathrm{BC} 1}^{\text {peak }}$, respectively. The accepted events have the beam impact point coordinates inside the square surface of the trigger scintillating counters:

$\left|x_{\mathrm{BC} 1}-x_{\mathrm{BC} 1}^{\mathrm{peak}}\right|<2.5 \mathrm{~cm}$

and

$\left|y_{\mathrm{BC} 1}-y_{\mathrm{BC} 1}^{\text {peak }}\right|<2.5 \mathrm{~cm}$.

The numbers of events retained after the application of this criterion are reported in Table 2 (Selection 2). 


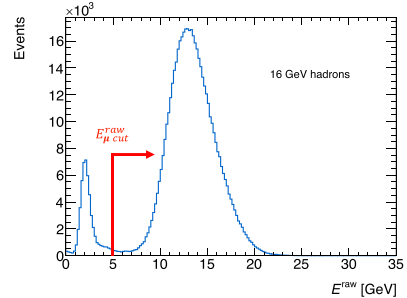

(a)

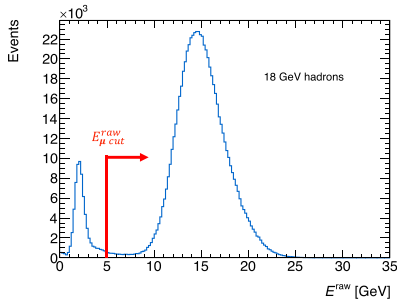

(b)
Fig. 5 Distributions of the energy $E^{\text {raw }}$ in $\mathrm{GeV}$ measured in the calorimeter modules in the case of particle beam energies equal to 16 $\mathrm{GeV}$ (a) and $18 \mathrm{GeV}$ (b). The events were selected applying the selection criteria up to Selection 2 (Table 2). The muons and spurious events were rejected in the analysis requiring $E^{\text {raw }}$ larger than $E_{\mu c u t}^{\text {raw }}=5 \mathrm{GeV}$, as shown in the histograms

\subsection{Identification of muons and electrons}

\subsubsection{Muon rejection}

A second set of criteria allows identifying pure samples of hadrons. As already mentioned, at the considered beam energies, muons are minimum ionizing particles and deposit in the scintillating tiles energy much smaller than electrons and hadrons (Fig. 5). The muon rejection was obtained requiring a reconstructed energy in the detector $E^{\text {raw }}$ (Sect. 2.2) larger than $E_{\mu c u t}^{\mathrm{raw}}=5 \mathrm{GeV}$. The selection criterion allows also a rejection of spurious trigger events. The retained events are reported in Table 2 (Selection 3).

\subsubsection{Electron identification}

As shown in Fig. 6, the signals $S_{\mathrm{Ch} 1}$ and $S_{\mathrm{Ch} 3}$ measured in the Cherenkov counters $\mathrm{Ch} 1$ and $\mathrm{Ch} 3$, respectively, allow a separation of pions and electrons $(e / \pi)$ from kaons $K$ and protons $p(K / p)$. The selection criteria in ADC counts applied on the signals are reported in Table 3 . The numbers of the identified events are reported in Table 2. As discussed in Sect. 3.3, the $\mathrm{Ch} 2$ measurements allow separating kaons and protons.

The electron components in $e / \pi$ samples were determined statistically exploiting the difference of electromagnetic and hadronic shower profiles in the calorimeter modules [12]. Two separators, $C_{\text {long }}$ and $C_{\mathrm{tot}}$, were used:

1. The shower profile parameter $C_{\text {long }}$ represents the fraction of the beam energy, $E_{\text {beam }}$, deposited in the layers A of the modules (Fig. 4) :

$$
C_{\text {long }}=\frac{\sum_{i=1}^{3} \sum_{j=1}^{3}\left(E_{\mathrm{c}}^{\mathrm{raw}}\right)_{i, j}}{E_{\mathrm{beam}}}
$$

where $i=1,2$ and 3 indicate the super-drawers M0 C, LBC65 and EBC65, respectively. The parameter $j$ runs over 3 contiguous cells of the three layers A around the
Table 3 Selection criteria in $S_{\mathrm{Ch} 1}, S_{\mathrm{Ch} 2}$ and $S_{\mathrm{Ch} 3}$ signals applied to identify $e / \pi, K$ and $p$ event samples for the four particle beam energy data sets. The Cherenkov signals are measured in ADC counts

\begin{tabular}{llll}
\hline$E_{\text {beam }}(\mathrm{GeV})$ & $e / \pi$ & $K$ & $p$ \\
\hline 16 & $S_{\mathrm{Ch} 1} \geq 500$ & $S_{\mathrm{Ch} 1}<500$ & $S_{\mathrm{Ch} 1}<500$ \\
& $S_{\mathrm{Ch} 3} \geq 470$ & $S_{\mathrm{Ch} 3} \leq 300$ & $S_{\mathrm{Ch} 3} \leq 300$ \\
& & $S_{\mathrm{Ch} 2} \geq 1500$ & $S_{\mathrm{Ch} 3} \leq 400$ \\
18 & $S_{\mathrm{Ch} 1} \geq 500$ & $S_{\mathrm{Ch} 1} \leq 500$ & $S_{\mathrm{Ch} 1} \leq 500$ \\
& $S_{\mathrm{Ch} 3} \geq 450$ & $S_{\mathrm{Ch} 3} \leq 400$ & $S_{\mathrm{Ch} 3} \leq 400$ \\
& & $S_{\mathrm{Ch} 2} \geq 2000$ & $S_{\mathrm{Ch} 3} \leq 400$ \\
& $S_{\mathrm{Ch} 1} \geq 500$ & $S_{\mathrm{Ch} 1} \leq 300$ & $S_{\mathrm{Ch} 1} \leq 300$ \\
& $S_{\mathrm{Ch} 3} \geq 450$ & $S_{\mathrm{Ch} 3} \leq 400$ & $S_{\mathrm{Ch} 3} \leq 400$ \\
30 & & $S_{\mathrm{Ch} 2} \geq 2000$ & $S_{\mathrm{Ch} 3} \leq 500$ \\
& $S_{\mathrm{Ch} 1} \geq 500$ & $S_{\mathrm{Ch} 1} \leq 400$ & $S_{\mathrm{Ch} 1} \leq 400$ \\
& $S_{\mathrm{Ch} 3} \geq 400$ & $S_{\mathrm{Ch} 3}<400$ & $S_{\mathrm{Ch} 3}<400$ \\
& & $S_{\mathrm{Ch} 2} \geq 1100$ & $S_{\mathrm{Ch} 3} \leq 200$ \\
\hline
\end{tabular}

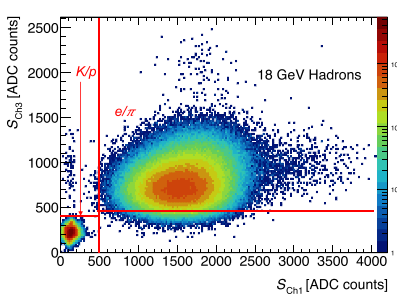

(a)

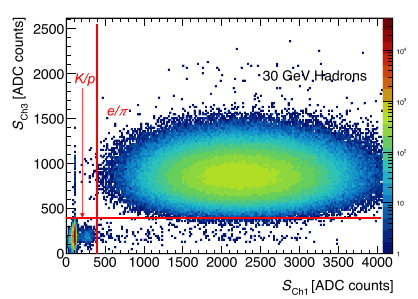

(b)
Fig. 6 Scatter plots of the signals measured in the Cherenkov counter $\mathrm{Ch} 3, S_{\mathrm{Ch} 3}$, in ADC counts as a function of the signals measured in the Cherenkov counter Ch1, $S_{\mathrm{Ch} 1}$, in ADC counts. The histograms were obtained analysing data with beam energies of $18 \mathrm{GeV}$ (a) and $30 \mathrm{GeV}$ (b). The events were selected applying selection criteria summarized in Table 2, up to Selection 3. The cut values used to select kaon and proton, $K / p$, (left/bottom) and electron and pion, $e / \pi$, (right/top) events are shown. The color scale indicates the number of events in the bins

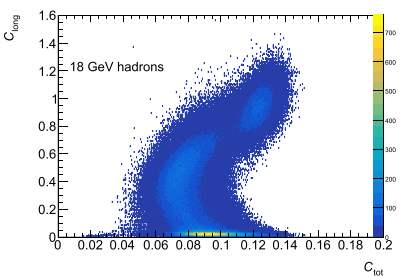

(a)

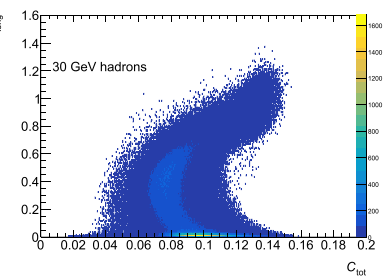

(b)
Fig. 7 Scatter plot $C_{\text {long }}$ vs $C_{\text {tot }}$ of $e / \pi$ sample events produced by beams of particles with energies equal to $18 \mathrm{GeV}$ (a) and $30 \mathrm{GeV}(\mathbf{b})$. The color scale indicates the number of events in the bins

cell hit by the beam and $E_{\mathrm{c}}^{\text {raw }}$ stands for the energy measured in a cell (Sect. 2.2).

2. The separator $C_{\text {tot }}$ measures the spread of the energy $E_{\mathrm{c}}^{\text {raw }}$ deposited in the cells of the modules:

$$
C_{\mathrm{tot}}=\frac{1}{\sum_{i=1}^{N_{\mathrm{cell}}}\left[\left(E_{\mathrm{c}}^{\mathrm{raw}}\right)_{i}\right]^{\alpha}}
$$




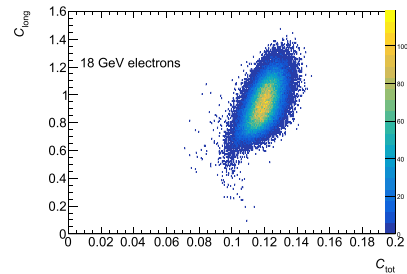

(a)

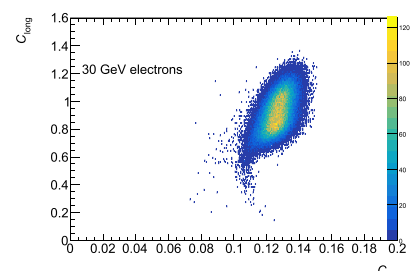

(c)

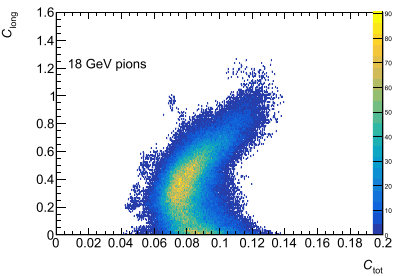

(b)

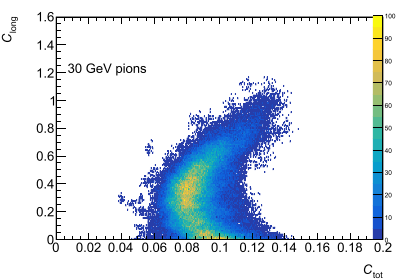

(d)
Fig. 8 Scatter plot $C_{\text {long }}$ vs $C_{\text {tot }}$ obtained using simulated $18 \mathrm{GeV}$ electrons (a), $18 \mathrm{GeV}$ pions (b), $30 \mathrm{GeV}$ electrons (c) and $30 \mathrm{GeV}$ pions (d) beams. The color scale indicate the number of events in the bins

$$
\times \sqrt{\frac{1}{N_{\text {cell }}} \sum_{i=1}^{N_{\text {cell }}}\left(\left[\left(E_{\mathrm{c}}^{\text {raw }}\right)_{i}\right]^{\alpha}-\frac{1}{N_{\text {cell }}} \sum_{i=1}^{N_{\text {cell }}}\left[\left(E_{\mathrm{c}}^{\text {raw }}\right)_{i}\right]^{\alpha}\right)^{2}}
$$

where $N_{\text {cell }}=24$ stands for the total number of contiguous cells, around the hit cell, considered for the shower profile estimate. The exponent $\alpha=0.6$ was tuned using a Monte Carlo (MC) simulation program to achieve maximum electron pion separation [12].

Scatter plots of $C_{\text {long }}$ vs $C_{\text {tot }}$ are shown in Fig. 7. The plots consist of $e / \pi$ sample events obtained using beams of particles with $E_{\text {beam }}$ equal to 18 and $30 \mathrm{GeV}$. They can be compared with the ones in Fig. 8 obtained using simulated electron and pion events with the same beam energies. Most pions have small values of $C_{\text {long }}$ and $\mathrm{C}_{\text {tot }}$. However pion events cause by showers with a large electromagnetic component have large $C_{\text {long }}$ and $\mathrm{C}_{\text {tot }}$ values. Electrons have parameters with larger values localized in narrower regions.

In addition electron $\mathrm{C}_{\text {tot }}$ distributions are well described by one Gaussian function while pion distributions require two Gaussian functions. As an example, Fig. 9a shows the experimental $\mathrm{C}_{\text {tot }}$ distribution obtained using an enriched electron beam with $E_{\text {beam }}=20 \mathrm{GeV}$ and $\mathrm{C}_{\text {long }} \geq \mathrm{C}_{\text {long }}^{\min }=0.6$. The fit was performed in the region $\mathrm{C}_{\text {tot }} \geq 1.125$. Figure $9 \mathrm{~b}$, c demonstrate that also simulated electron $\mathrm{C}_{\text {tot }}$ distributions at 18 and $30 \mathrm{GeV}$ are well described by one Gaussian function.

Pion $C_{\mathrm{tot}}$ distributions are best described by two Gaussian functions. The distributions of $e / \pi$ data events with $\mathrm{C}_{\text {long }}<$ $\mathrm{C}_{\text {long }}^{\min }=0.6$ and $E_{\text {beam }}$ equal to 18 and $30 \mathrm{GeV}$, respectively, are shown in Fig. 10. It displays the fit by sum of two Gaussian functions as well as the individual Gaussian functions used to produce the fit. In Fig. $11 C_{\text {tot }}$ distributions of simulated

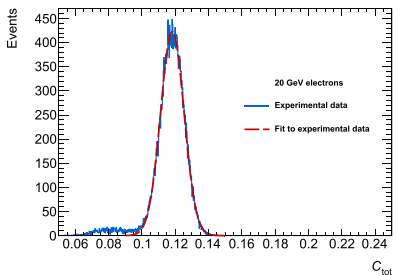

(a)

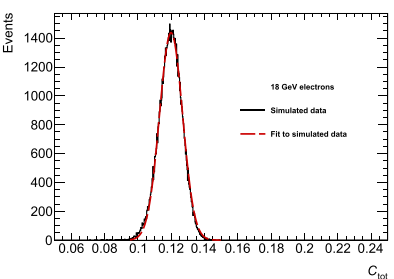

(b)

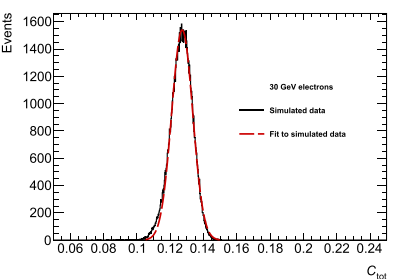

(c)
Fig. 9 Distributions of $C_{\text {tot }}$ obtained using experimental electronenriched beam particles with an energy equal to $20 \mathrm{GeV}$ (a) and simulated electrons with $E_{\text {beam }}$ equal to $18 \mathrm{GeV}$ (b) and $30 \mathrm{GeV}$ (c). Fit Gaussian functions obtained using the method of the least squares are superimposed in red on the distributions

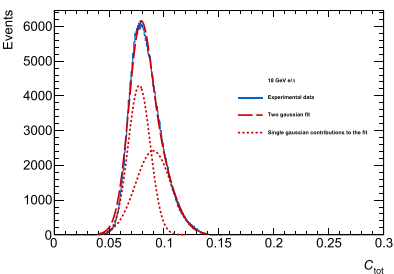

(a)

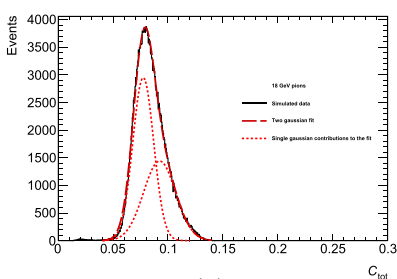

(c)

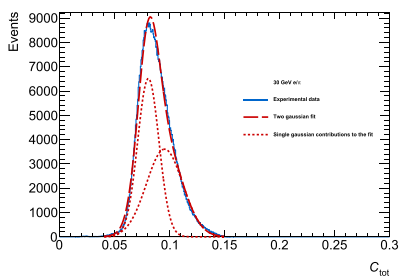

(b)

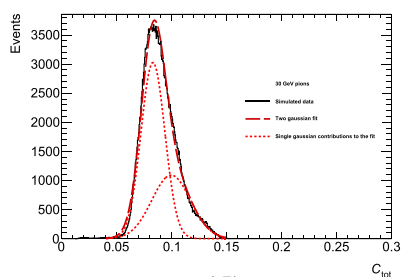

(d)
Fig. 10 The blue histograms in $\mathbf{a}$ and $\mathbf{b}$ show $C_{\text {tot }}$ distributions of experimental $e / \pi$ sample events with $E_{\text {beam }}$ equal to $18 \mathrm{GeV}$ and 30 $\mathrm{GeV}$, respectively. Samples of pion events were selected requiring $\mathrm{C}_{\text {long }}$ $<0.6$. The black histograms $\mathbf{c}$ and $\mathbf{d}$ show the same distributions for simulated pion events selected applying the same selection criteria and with $\mathrm{E}_{\text {beam }}$ equal to $18 \mathrm{GeV}$ and $30 \mathrm{GeV}$, respectively. Two Gaussian functions fits, obtained using the method of the least squares, are overlapped to the data (red dashed curves). Red dotted curves show the individual Gaussian contributions

pions with $\mathrm{C}_{\text {long }} \geq \mathrm{C}_{\text {long }}^{\min }=0.6$ and $\mathrm{E}_{\text {beam }}$ equal to $18 \mathrm{GeV}$ (a) and $30 \mathrm{GeV}$ (b) are shown. They are also well described by the sum of two Gaussian functions.

The number of electrons in the four $e / \pi$ samples were determined considering $\mathrm{C}_{\text {tot }}$ distributions of the events with $\mathrm{C}_{\text {long }} \geq \mathrm{C}_{\text {long }}^{\min }=0.6$. Examples of such distributions obtained in the case of events produced by beams of par- 

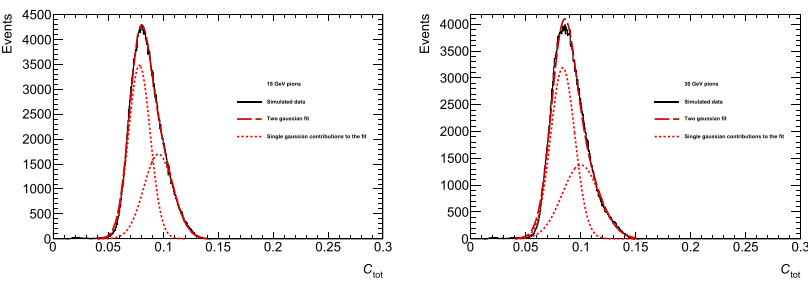

Fig. 11 The black histograms show $C_{\text {tot }}$ distributions obtained using simulated pions with $\mathrm{E}_{\text {beam }}$ equal to $18 \mathrm{GeV}$ (a) and $30 \mathrm{GeV}$ (b). The events were selected requiring $\mathrm{C}_{\text {long }} \geq 0.6$. Two Gaussian functions fits, obtained using the method of the least squares, are superimposed on the data (red dashed curve). Red dotted curves show the individual Gaussian contributions

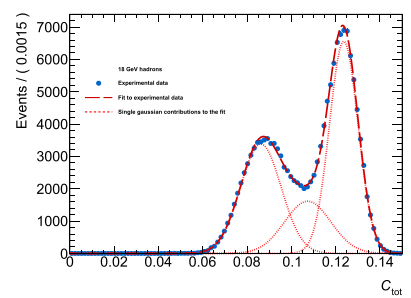

(a)

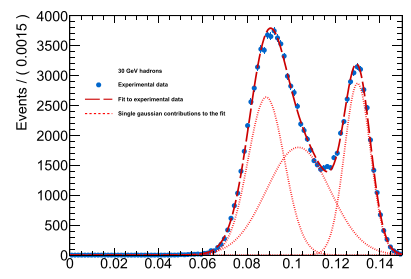

(c)

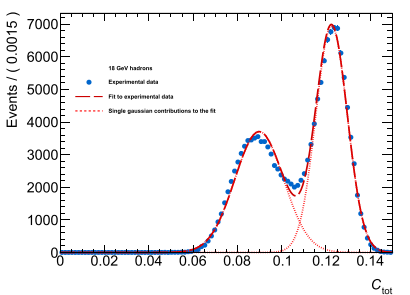

(b)

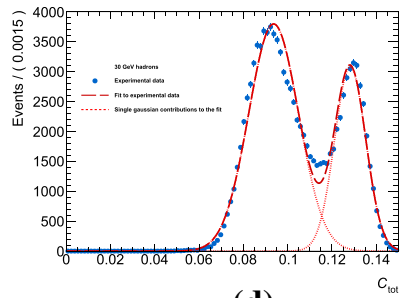

(d)
Fig. 12 The blue dotted histograms represent the $C_{\text {tot }}$ distributions of $e / \pi$ sample events with $E_{\text {beam }}$ equal to $18 \mathrm{GeV}(\mathbf{a}, \mathbf{b})$ and $30 \mathrm{GeV}(\mathbf{c}, \mathbf{d})$, respectively. The events are limited to those with $\mathrm{C}_{\text {long }} \geq 0.6$. Multimodal three- and two-Gaussian fit functions (red dashed curves) are superimposed on the histograms (a, $\mathbf{c}$ and $\mathbf{b}, \mathbf{d})$, respectively. The functions parameters were obtained using the maximum likelihood method. Red dotted curves show the individual Gaussian function contributions to these fits. In each histogram, the function with the largest value of the mean $\mu$ describes the electron contamination. As discussed in Sect. 3.2.2, Three-Gaussian fits were used to determine the numbers of electrons contaminating the samples. Two-Gaussian fits were used to estimate uncertainties on the determinations (Sect. 5.1)

ticle with energies equal to $18 \mathrm{GeV}$ and $30 \mathrm{GeV}$ are shown in Fig. 12. Three Gaussian functions were fitted to the experimental distributions using the maximum likelihood method. The fit functions are superimposed on the histograms in Fig. 12a, c. The individual Gaussian function contributions are also shown. The functions with the largest mean values $\mu$ describe the electron contributions. The numbers of the electrons reported in Table 2 are determined from the areas limited by such functions. The statistical uncertainties are equal to the corresponding diagonal terms of the fit error matrices. The estimated percentage of electrons increases from a value of $11 \%$ at $16 \mathrm{GeV}$ up to $28 \%$ at $30 \mathrm{GeV}$.

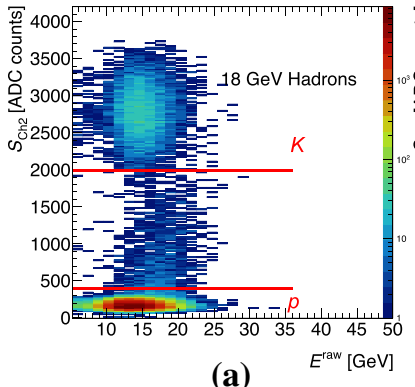

(a)

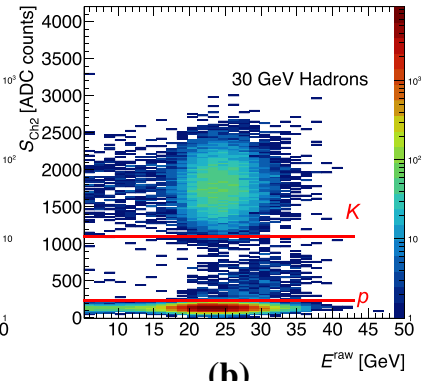

(b)
Fig. 13 Scatter plot of the $\mathrm{Ch} 2$ signals, $S_{\mathrm{Ch} 2}$, in $\mathrm{ADC}$ counts units, vs the energy measured in the calorimeter $E^{\text {raw }}$ obtained by analyzing $K / p$ sample events produced by beams of particles with energy equal to 18 (a) and 30 (b) GeV. The cut values applied in the analysis to select kaon and proton events are shown. The color scale indicates the number of events in the bins

\subsection{Pion, Kaon and Proton identification}

The third set of selection criteria was specific to the type of hadronic particles being studied. For each $E_{\text {beam }}$ data set the number of pions reported in Table 2 was estimated by subtracting the number of electron events obtained using the method described in Sect. 3.2.2 from the number of events of the corresponding $e / \pi$ sample. The $\mathrm{Ch} 2$ signal measurements allow a separation of kaons and protons in the $K / p$ samples. The scatter plots of the $\mathrm{Ch} 2$ signals, $S_{\mathrm{Ch} 2}$, in ADC counts units vs the energy measured in the calorimeter, $E^{\text {raw }}$, obtained by analyzing data produced by beams of particles with energies equal to 18 and $30 \mathrm{GeV}$, are shown in Fig. 13 . The $S_{\mathrm{Ch} 2}$ selection values in ADC count units are reported in Table 3. The obtained numbers of kaons and protons are reported in Table 2 .

\subsection{Reconstruction of the energy deposited in the modules}

As already discussed in Sect. 2.2, the energy $E^{\text {raw }}$ deposited by incident particles in the detector was obtained as the sum of the energy measured in the calorimeter cells. In this study only cells with $\left|E_{\mathrm{c}}^{\text {raw }}\right|>2 \sigma_{\text {noise }}$ were considered in the sum. For each run, the cell electronics noise $\sigma_{\text {noise }}$ was determined using random events collected between beam bursts. Typical noise values are about $30 \mathrm{MeV}$. No corrections for dead material, containment and non-compensation effects were applied.

To account for electron contamination, as sketched in the Fig. 14, the pion energy distributions $n_{\pi}\left(E^{\text {raw }}\right)$ were obtained using, bin per bin, the formula

$n_{\pi}\left(E^{\mathrm{raw}}\right)=n_{e / \pi}\left(E^{\mathrm{raw}}\right)-N_{e} f_{e}\left(E^{\mathrm{raw}}\right)$,

where $n_{e} / \pi\left(E^{\mathrm{raw}}\right)$ is the number of $e / \pi$ events in the considered $E^{\text {raw }}$ bin, the electron distribution $f_{e}\left(E^{\text {raw }}\right)$ is normalized to 1 and the number of electrons, $N_{e}$, was determined 


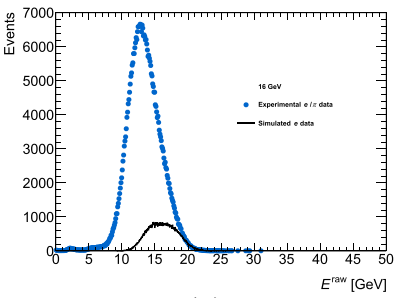

(a)

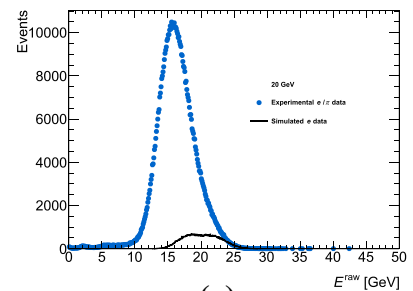

(c)

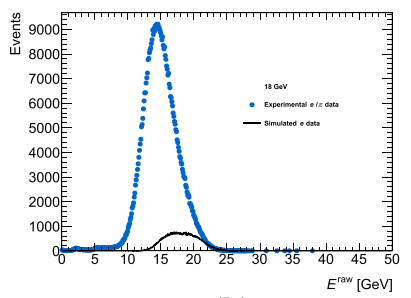

(b)

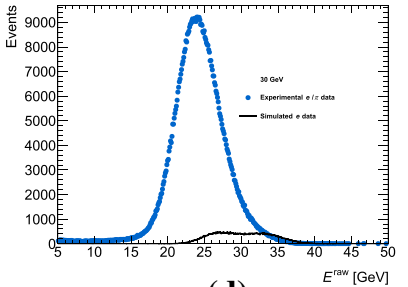

(d)

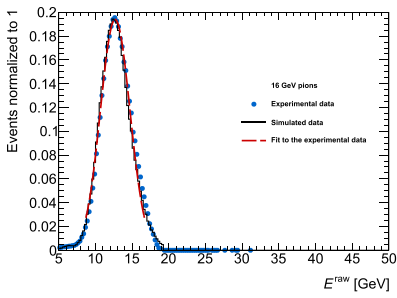

(a)

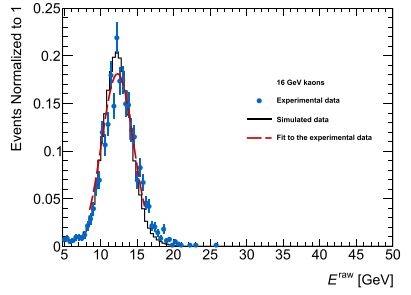

(b)

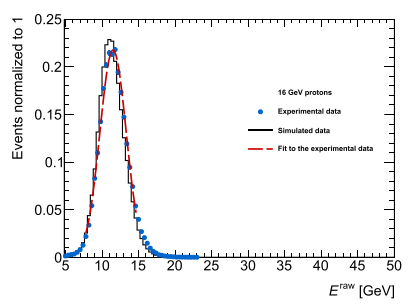

(c)

Fig. 14 The blue dotted histograms show the experimental data distributions of the reconstructed energy $E^{\text {raw }}$ of the $e / \pi$ samples events with $E_{\text {beam }}$ equal to $16 \mathrm{GeV}$ (a), $18 \mathrm{GeV}(\mathbf{b}), 20 \mathrm{GeV}$ (c) and $30 \mathrm{GeV}$ (d). The black dotted histograms, obtained using simulated events, correspond to the expected distributions of electrons contaminating the samples. The normalization procedure is described in Sect. 3.4

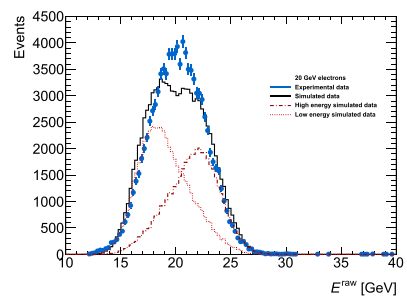

(a)

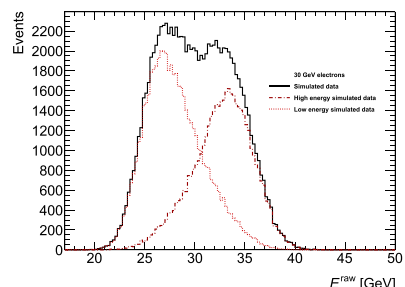

(c)

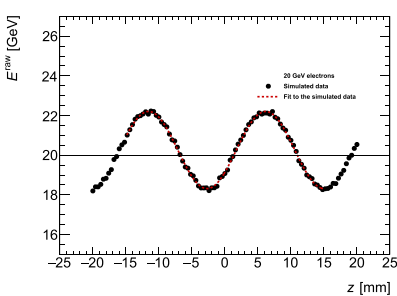

(b)

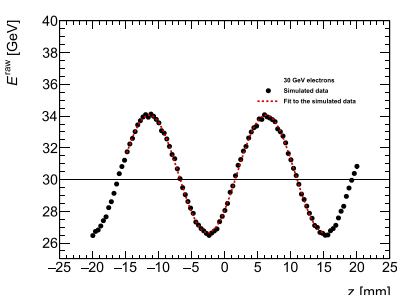

(d)
Fig. 15 The black histograms in a and $\mathbf{c}$ show the distributions of the reconstructed energy $E^{\text {raw }}$ obtained using simulated electron beams with $E_{\text {beam }}$ equal to $20 \mathrm{GeV}$ and $30 \mathrm{GeV}$, respectively. The blue dot distribution in a has been obtained using experimental data (Sect. 3.4). The distributions obtained using "high energy events" (red dashed line) and "low energy events" (red dotted line) discussed in Sect. 5.1 are shown in (a) and (c). The histograms (b) and $\mathbf{d}$ show the oscillation of the electron response due to the sampling fraction variations as obtained using simulated electrons with $E_{\text {beam }}$ equal to $20 \mathrm{GeV}$ and $30 \mathrm{GeV}$, respectively (Sect. 5.1). The dashed curves in red correspond to the fit of Eq. 10 to the data. The horizontal black line corresponds to the electron mean energy parameter $p_{0}$
Fig. 16 Distributions of the reconstructed energy $E^{\text {raw }}$ obtained analyzing pion (a), kaon (b) and proton (c) data with $E_{\text {beam }}=16 \mathrm{GeV}$. The blue dotted histograms represent the experimental data. Only statistical uncertainties are shown. The dashed curves in red correspond to the fit of a Gaussian function to the experimental data in a region $\pm 2 \sigma$ around the peak value. The black histograms correspond to the predictions of the MC simulation

Table 4 Numbers of simulated (generated events) and retained pion, kaon and proton events for each beam energy value. Selection criteria used in the analysis are discussed in Sect. 4

\begin{tabular}{lllll}
\hline$E_{\text {beam }}[\mathrm{GeV}]$ & 16 & 18 & 20 & 30 \\
\hline Generated Events & \multicolumn{5}{c}{300000} \\
Pions & 283222 & 285211 & 286574 & 291040 \\
Kaons & 247559 & 253040 & 256514 & 269728 \\
Protons & 292412 & 293891 & 294596 & 296532 \\
\hline
\end{tabular}

using the procedure described in Sect. 3.2.2. Simulated electron distributions were used in the analysis because experimental data are available only for electron beam energy equal to $20 \mathrm{GeV}$. The distributions of the reconstructed energy $E^{\text {raw }}$ obtained using simulated electron beams with $E_{\text {beam }}$ equal to $20 \mathrm{GeV}$ and $30 \mathrm{GeV}$ are shown in Fig. 15. The $20 \mathrm{GeV}$ experimental and simulated $E^{\text {raw }}$ distributions are compared in Fig. 15a. The experimental data spread is slightly smaller than the the simulated data one due to slightly larger incident angle of the experimental beam with respect to the normal of the calorimeter cell surface. This difference also explains the washing out in the experimental distribution of the double peak structure appearing in the simulated data.

Figures $16,17,18$ and 19 show the $E^{\text {raw }}$ distributions of pions, kaons and protons for beam energies of $16,18,20$, and $30 \mathrm{GeV}$. 


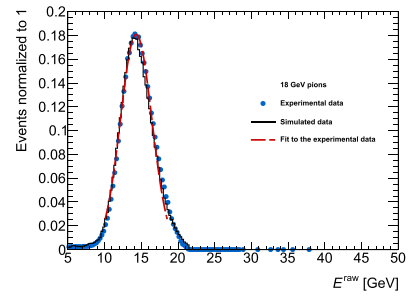

(a)

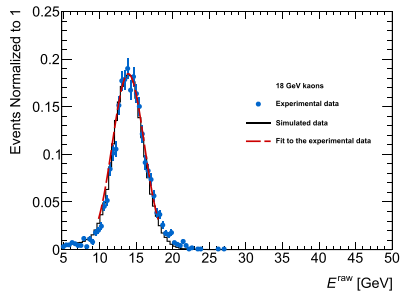

(b)

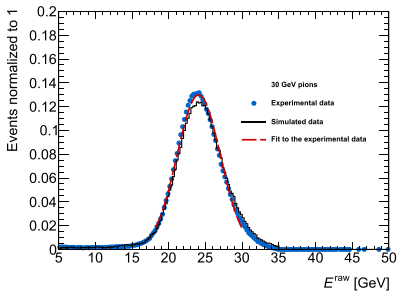

(a)

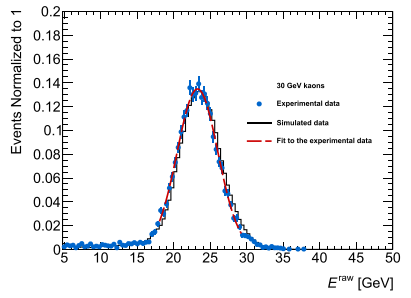

(b)

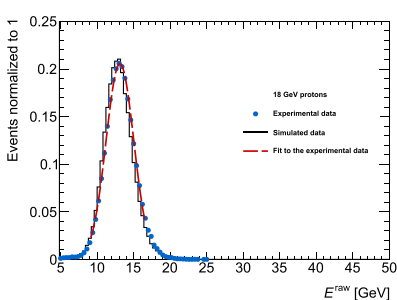

(c)

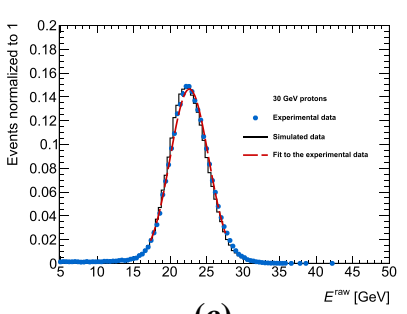

(c)
Fig. 17 Distributions of the reconstructed energy $E^{\text {raw }}$ obtained analyzing pion (a), kaon (b) and proton (c) data with $E_{\text {beam }}=18 \mathrm{GeV}$. The blue dotted histograms represent the experimental data. Only statistical uncertainties are shown. The dashed curves in red correspond to the fit of a Gaussian function to the experimental data in a region $\pm 2 \sigma$ around the peak value. The black histograms correspond to the predictions of the MC simulation

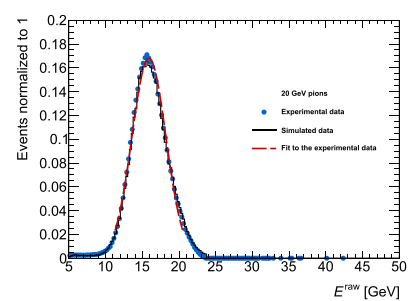

(a)

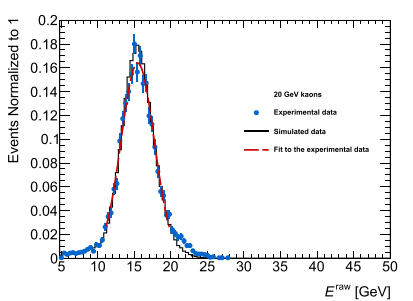

(b)

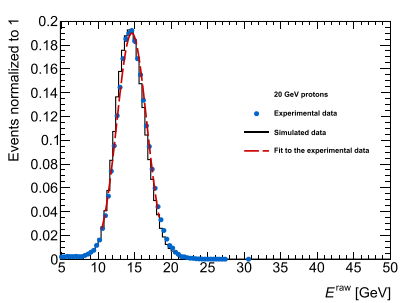

(c)

Fig. 18 Distributions of the reconstructed energy $E^{\text {raw }}$ obtained analyzing pion (a), kaon (b) and proton (c) data with $E_{\text {beam }}=20 \mathrm{GeV}$. The blue dotted histograms represent the experimental data. Only statistical uncertainties are shown. The dashed curves in red correspond to the fit of a Gaussian function to the experimental data in a region $\pm 2 \sigma$ around the peak value. The black histograms correspond to the predictions of the MC simulation
Fig. 19 Distributions of the reconstructed energy $E^{\text {raw }}$ obtained analyzing pion (a), kaon (b) and proton (c) data with $E_{\text {beam }}=30 \mathrm{GeV}$. The blue dotted histograms represent the experimental data. Only statistical uncertainties are shown. The dashed curves in red correspond to the fit of a Gaussian function to the experimental data in a region $\pm 2 \sigma$ around the peak value. The black histograms correspond to the predictions of the MC simulation

\section{Analysis of simulated data}

The experimental results obtained using positive pions and kaons, and protons beams with energies in the range 16-30 $\mathrm{GeV}$, were compared to the predictions of the Geant4-based ATLAS simulation program [3-5]. The FTFP_BERT_ATL hadronic showering model [14] was used in the simulation. This model is presently being used in the simulation of the ATLAS events collected during the Run 1 and Run 2 of the LHC. The numbers of pion, kaon and proton generated events for each value of $E_{\text {beam }}$ are reported in Table 4 . The responses of the beam line detectors were not included in the simulation. The distributions of the transverse beam impact point coordinates in the detector were tuned to reproduce the ones measured using the $\mathrm{BC}$. The $\mathrm{TB}$ detector material and geometry are fully described [4]. The measured electronics noise in the different calorimeter cells and the effects of photo-statistics in the PMT signals (70 photo-electrons/GeV) are included in the $\mathrm{MC}$ simulation. The simulated pion events were selected applying the $C_{\text {long }}$ and $C_{\text {tot }}$ cuts used in the analysis of experimental data. The numbers of the retained events for analyses are reported in Table 4 . The shower energy was reconstructed using the same procedure applied in the case of experimental data. The distributions of $E^{\text {raw }}$ obtained using simulated data are shown in Figs. 16, 17, 18 and 19 for beam energies equal to $16,18,20$ and $30 \mathrm{GeV}$, respectively. 
Table 5 Energy responses $\left(\left\langle E^{\text {raw }}\right\rangle\right)$ and resolutions $\left(\sigma^{\text {raw }}\right)$ obtained fitting Gaussian functions to the experimental and simulated $E^{\text {raw }}$ distributions of pions $(\pi)$, kaons $(K)$ and protons $(p)$ with different beam energies. Uncertainties correspond to statistical uncertainties on the fit parameters

\begin{tabular}{|c|c|c|}
\hline$E_{\text {beam }}(\mathrm{GeV})$ & Exp. data & Sim. data \\
\hline \multicolumn{3}{|c|}{$\left\langle E^{\mathrm{raw}}\right\rangle(\pi)$} \\
\hline 16 & $12.678 \pm 0.008$ & $12.500 \pm 0.008$ \\
\hline 18 & $14.294 \pm 0.007$ & $14.134 \pm 0.009$ \\
\hline 20 & $15.896 \pm 0.004$ & $15.744 \pm 0.010$ \\
\hline 30 & $24.058 \pm 0.004$ & $24.110 \pm 0.013$ \\
\hline \multicolumn{3}{|c|}{$\sigma^{\text {raw }}(\pi)$} \\
\hline 16 & $2.013 \pm 0.014$ & $1.948 \pm 0.015$ \\
\hline 18 & $2.139 \pm 0.012$ & $2.122 \pm 0.017$ \\
\hline 20 & $2.319 \pm 0.004$ & $2.289 \pm 0.019$ \\
\hline 30 & $2.962 \pm 0.004$ & $2.966 \pm 0.026$ \\
\hline \multicolumn{3}{|c|}{$\left\langle E^{\text {raw }}\right\rangle(K)$} \\
\hline 16 & $12.291 \pm 0.252$ & $12.236 \pm 0.004$ \\
\hline 18 & $13.886 \pm 0.114$ & $13.899 \pm 0.005$ \\
\hline 20 & $15.445 \pm 0.034$ & $15.459 \pm 0.005$ \\
\hline 30 & $23.244 \pm 0.035$ & $23.636 \pm 0.006$ \\
\hline \multicolumn{3}{|c|}{$\sigma^{\mathrm{raw}}(K)$} \\
\hline 16 & $2.168 \pm 0.441$ & $1.904 \pm 0.004$ \\
\hline 18 & $2.175 \pm 0.226$ & $2.059 \pm 0.005$ \\
\hline 20 & $2.262 \pm 0.034$ & $2.183 \pm 0.005$ \\
\hline 30 & $2.790 \pm 0.035$ & $2.831 \pm 0.006$ \\
\hline \multicolumn{3}{|c|}{$\left\langle E^{\mathrm{raw}}\right\rangle(p)$} \\
\hline 16 & $11.511 \pm 0.008$ & $11.234 \pm 0.003$ \\
\hline 18 & $13.119 \pm 0.007$ & $12.827 \pm 0.004$ \\
\hline 20 & $14.606 \pm 0.006$ & $14.429 \pm 0.004$ \\
\hline 30 & $22.649 \pm 0.009$ & $22.457 \pm 0.005$ \\
\hline \multicolumn{3}{|c|}{$\sigma^{\text {raw }}(p)$} \\
\hline 16 & $1.795 \pm 0.007$ & $1.729 \pm 0.004$ \\
\hline 18 & $1.898 \pm 0.006$ & $1.854 \pm 0.004$ \\
\hline 20 & $2.047 \pm 0.006$ & $1.989 \pm 0.004$ \\
\hline 30 & $2.633 \pm 0.009$ & $2.588 \pm 0.005$ \\
\hline
\end{tabular}

\section{Determination of the energy response and resolution}

The $E^{\text {raw }}$ distributions of pion, kaon and proton data are described reasonably well around the peak values by a Gaussian function. As in [12], the $\mu$ and $\sigma$ parameters of Gaussian functions fitting the distributions in a region $\pm 2 \sigma$ around the peak values were used to estimate the measurement responses $\left\langle E^{\text {raw }}\right\rangle$ and resolutions $\sigma^{\text {raw }}$. An iterative procedure, using the method of least squares, has been applied in order to get stable values of the parameters. The fit functions obtained analysing experimental data are superimposed over the corresponding distributions in Figs. 16, 17, 18 and 19. The fit results obtained using experimental and simulated data are
Table 6 Energy responses $\left(R^{\left\langle E^{\text {raw }}\right\rangle}\right)$ and resolutions $\left(R^{\sigma^{\text {raw }}}\right)$ normalized to incident beam energy obtained using pion $(\pi)$, kaon $(K)$ and proton $(p)$ data with different beam energies. In the case of experimental data, statistical and systematic uncertainties are reported. The effects of the different systematic uncertainty sources, discussed in Sect. 5.1, were combined in quadrature. Only statistical uncertainties are reported for simulated data

\begin{tabular}{|c|c|c|}
\hline$E_{\text {beam }}(\mathrm{GeV})$ & Exp. Data & Sim. Data \\
\hline \multicolumn{3}{|c|}{$R^{\left\langle E^{\mathrm{raw}}\right\rangle}(\pi)$} \\
\hline 16 & $0.7924 \pm 0.0005 \pm 0.0116$ & $0.7812 \pm 0.0005$ \\
\hline 18 & $0.7941 \pm 0.0004 \pm 0.0108$ & $0.7852 \pm 0.0005$ \\
\hline 20 & $0.7948 \pm 0.0002 \pm 0.0101$ & $0.7872 \pm 0.0005$ \\
\hline 30 & $0.8019 \pm 0.0001 \pm 0.0098$ & $0.8036 \pm 0.0004$ \\
\hline \multicolumn{3}{|c|}{$R^{\sigma^{\mathrm{raw}}}(\pi)$} \\
\hline 16 & $0.1258 \pm 0.0009 \pm 0.0038$ & $0.1217 \pm 0.0009$ \\
\hline 18 & $0.1188 \pm 0.0007 \pm 0.0022$ & $0.1179 \pm 0.0009$ \\
\hline 20 & $0.1159 \pm 0.0002 \pm 0.0013$ & $0.1144 \pm 0.0010$ \\
\hline 30 & $0.0987 \pm 0.0001 \pm 0.0006$ & $0.0988 \pm 0.0008$ \\
\hline \multicolumn{3}{|c|}{$R^{\left\langle E^{\mathrm{raw}}\right\rangle}(K)$} \\
\hline 16 & $0.7682 \pm 0.0158 \pm 0.0094$ & $0.7647 \pm 0.0003$ \\
\hline 18 & $0.7714 \pm 0.0064 \pm 0.0093$ & $0.7721 \pm 0.0003$ \\
\hline 20 & $0.7723 \pm 0.0017 \pm 0.0093$ & $0.7729 \pm 0.0002$ \\
\hline 30 & $0.7748 \pm 0.0012 \pm 0.0093$ & $0.7878 \pm 0.0002$ \\
\hline \multicolumn{3}{|c|}{$R^{\sigma^{\text {raw }}}(K)$} \\
\hline 16 & $0.1356 \pm 0.0276 \pm 0.000007$ & $0.1190 \pm 0.0003$ \\
\hline 18 & $0.1209 \pm 0.0126 \pm 0.0005$ & $0.1144 \pm 0.0002$ \\
\hline 20 & $0.1131 \pm 0.0017 \pm 0.0008$ & $0.1091 \pm 0.0002$ \\
\hline 30 & $0.0930 \pm 0.0012 \pm 0.0002$ & $0.0943 \pm 0.0002$ \\
\hline \multicolumn{3}{|c|}{$R^{\left\langle E^{\mathrm{raw}}\right\rangle}(p)$} \\
\hline 16 & $0.7195 \pm 0.0005 \pm 0.0086$ & $0.7021 \pm 0.0002$ \\
\hline 18 & $0.7288 \pm 0.0004 \pm 0.0087$ & $0.7126 \pm 0.0002$ \\
\hline 20 & $0.7303 \pm 0.0003 \pm 0.0088$ & $0.7214 \pm 0.0002$ \\
\hline 30 & $0.7549 \pm 0.0003 \pm 0.0091$ & $0.7485 \pm 0.0001$ \\
\hline \multicolumn{3}{|c|}{$R^{\sigma^{\text {raw }}}(p)$} \\
\hline 16 & $0.1122 \pm 0.0004 \pm 0.000034$ & $0.1081 \pm 0.0002$ \\
\hline 18 & $0.1055 \pm 0.0003 \pm 0.0004$ & $0.1030 \pm 0.0002$ \\
\hline 20 & $0.1024 \pm 0.0003 \pm 0.0007$ & $0.0994 \pm 0.0002$ \\
\hline 30 & $0.0877 \pm 0.0003 \pm 0.0002$ & $0.0862 \pm 0.0001$ \\
\hline
\end{tabular}

reported in Table 5. The statistical uncertainties correspond to the square root of the corresponding diagonal term of the fit error matrix.

5.1 Energy responses and resolutions normalized to incident beam energy

Energy response normalized to incident beam energy

$R^{\left\langle E^{\text {raw }}\right\rangle}=\frac{\left\langle E^{\text {raw }}\right\rangle}{E_{\text {beam }}}$ 
and energy resolution normalized to incident beam energy

$R^{\sigma^{\text {raw }}}=\frac{\sigma^{\text {raw }}}{E_{\text {beam }}}$

obtained for the different values of $E_{\text {beam }}$ are reported in Table 6 . In the case of simulated data only statistical uncertainties are reported. In the case of experimental results, the first uncertainty value corresponds to the statistical uncertainty. The second value, describing the systematic uncertainty, was obtained considering the following seven sources:

1. The first source (Source 1), affecting only pion determinations, is the amount of electrons contaminating the $e / \pi$ samples. The systematic uncertainties on (8) and (9) reported in Table 7 correspond to the statistical uncertainties of the determinations of the number of electrons discussed in Sect. 3.2.2.

2. As discussed in the same section, the electron contamination was determined studying the $\mathrm{C}_{\text {tot }}$ distributions of the $e / \pi$ sample events with $\mathrm{C}_{\text {long }} \geq \mathrm{C}_{\text {long }}^{\min }=0.6$. Source 2 uncertainty values reported in Table 7 correspond to half of the differences of the determinations of $R^{\left\langle E^{\text {raw }}\right\rangle}$ and $R^{\sigma^{\text {raw }}}$ obtained using $\mathrm{C}_{\text {long }}^{\text {min }}=0.5$ and $\mathrm{C}_{\text {long }}^{\text {min }}=0.7$.

3. Effects due to the miss-modeling of the $C_{\text {tot }}$ distributions used to determine the number of contaminating electrons were estimated comparing the results obtained using three Gaussian functions fits (Sect. 3.2.2) with the ones obtained using two Gaussian functions fits. Source 3 uncertainty values, affecting only pion determinations, reported in Table 7 for each of the four beam energy samples, are equal to the differences of the values of $R^{\left\langle E^{\text {raw }}\right\rangle}$ and $R^{\sigma^{\text {raw }}}$ obtained using the two fitting functions.

4. As discussed in Sect. 3.4 the experimental $E^{\text {raw }}$ distributions of pions were obtained by using simulated electrons $E^{\text {raw }}$ distributions (Eq. (7)). Due to the regularly spaced scintillating tiles (Fig. 3) and the compactness of electromagnetic showers, the electron response varies with the periodicity of sampling fraction and thus, for a given incident angle, depends on the coordinate of the impact point of the beam particles along the front face of the calorimeter module $(z)$. Fig. 15b, d show that the variation is reasonably well described by a simple periodic function [12]

$E^{\mathrm{raw}}(z)=p_{0}\left[1+p_{1} \sin \left(2 \pi z / p_{2}\right)+p_{3}\right]$.

The parameter $p_{0}$ corresponds to the mean reconstructed energy. The relative amplitude of the oscillation is described by $p_{1}$. The parameter $p_{2}$ corresponds to the periodic thickness as seen by the beam at a given $z$ value and $p_{3}$ is a phase. The behavior is responsible of the two peak structure of the $E^{\text {raw }}$ distributions evident, in par- ticular, in the case of $E_{\text {beam }}=30 \mathrm{GeV}$ simulated data in Fig. 15c. The effects of the uncertainty on the distribution of the $z$ coordinates of the electron impact point on the determinations of $R^{\left\langle E^{\text {raw }}\right\rangle}$ and $R^{\sigma^{\text {raw }}}$ was estimated using the $E^{\text {raw }}$ distributions of the events with a $z$ value corresponding to $E^{\text {raw }}>p_{0}$, "high energy events", and $E^{\text {raw }}$ $<p_{0}$, "low energy events". The distributions are shown in Fig. 15a, c. Source 4 systematic uncertainty values, reported in Table 7, are derived from half of the differences of the values obtained using the two distributions. This uncertainty affects only pion determinations.

5. The $30 \mathrm{GeV}$ scatter plot of $S_{\mathrm{Ch} 1}$ vs. $S_{\mathrm{Ch} 3}$ in Fig. 6 shows two high-density regions in the $K / p$ region. Their origin is not clear. Source 5 systematic uncertainty values reported in Table 7 correspond to the differences of the values of $R^{\left\langle E^{\text {raw }}\right\rangle}$ and $R^{\sigma^{\text {raw }}}$ obtained using the events with $S_{\mathrm{Ch} 1} \leq 400$ ADC counts and $S_{\mathrm{Ch} 1} \leq 250 \mathrm{ADC}$ counts. Although the other three energy data points do not show the two spot structure, a systematic uncertainty was also determined for them using the described procedure with the same selection criterion values. In the case of $E_{\text {beam }}$ equal to $16 \mathrm{GeV}$, the estimated uncertainties on $R^{\left\langle E^{\text {raw }}\right\rangle}(p)$ and $R^{\sigma^{\text {raw }}}(K)$ were found to be negligible.

6. As shown in Fig. 13, proton $S_{\mathrm{Ch} 2}$ distributions exhibit large tails whose origin is not understood. For each of the four proton beam energies, Source 6 systematic uncertainty values in Table 7, correspond to the differences between the values of $R^{\left\langle E^{\text {raw }}\right\rangle}$ and $R^{\sigma^{\text {raw }}}$ obtained using the upper values of the $S_{\mathrm{Ch} 2}$ signals of Table 3, and the ones obtained selecting the events with $S_{\mathrm{Ch} 2}$ smaller than 2000 ADC counts at $16 \mathrm{GeV}, 18 \mathrm{GeV}$ and $20 \mathrm{GeV}$ and $1000 \mathrm{ADC}$ counts at $30 \mathrm{GeV}$, respectively. The same effect could be present also in the case of kaons, where the signal would fall in the low value tails of the $\mathrm{Ch} 2 \mathrm{sig}$ nals. For this reason, the systematic uncertainty obtained for protons is applied in kaon determinations too.

7. The effect of the uncertainty of the scale of the reconstructed cell energy $\Delta C_{\mathrm{c}}^{\mathrm{EM}}$ on the measurements was also investigated. An estimation of the uncertainty on the energy response can be obtained using the formula:

$$
\Delta\left\langle E^{\mathrm{raw}}\right\rangle^{\mathrm{EM}}=\Delta C_{\mathrm{c}}^{\mathrm{EM}} \sqrt{\sum_{i}\left\langle E_{c}^{\mathrm{raw}}\right\rangle_{i}^{2}}
$$

where $\Delta C_{\mathrm{c}}^{\mathrm{EM}}$ is equal to $2.4 \%$ (Sect. 2.2) and $\left\langle E_{c}^{\mathrm{raw}}\right\rangle_{i}$ is the average energy deposited in the cell $i$. The beam energies are known at few per mile and one obtains the values of $\Delta R^{\left\langle E^{\text {raw }}\right\rangle}$ reported in Table 7, Source 7, for the twelve data points. No significant dependence of the values on the beam energy values was found. The uncertainty on $C_{\mathrm{c}}^{\mathrm{EM}}$ affects in a negligible way the determinations of $R^{\sigma^{\text {raw }}}$. 
Table 7 Systematic uncertainties on the estimations of $R^{\left\langle E^{\text {raw }}\right\rangle}$ and $R^{\sigma^{\text {raw }}}$ in percent. The pion measurements are affected by the uncertainty on the number of electrons contaminating the $e / \pi$ samples (Sources 1,2 and 3), on the $E^{\text {raw }}$ shape of the contaminating electrons (Source 4). The kaon and proton measurements are affected by the uncertainty on the Ch1 (Source 5) and Ch2 (Source 6) selection criteria. The uncertainty on the determination of the cell energy response non-uniformity, Source 7 , affects the determinations obtained for the three particle beams

\begin{tabular}{|c|c|c|c|c|c|c|c|c|c|}
\hline \multicolumn{2}{|c|}{$E_{\text {beam }}(\mathrm{GeV})$} & \multicolumn{2}{|l|}{16} & \multicolumn{2}{|l|}{18} & \multicolumn{2}{|l|}{20} & \multicolumn{2}{|l|}{30} \\
\hline Source & Beam part. & $R^{\left\langle E^{\text {raw }}\right\rangle}(\%)$ & $R^{\sigma^{\text {raw }}}(\%)$ & $R^{\left\langle E^{\text {raw }}\right\rangle}(\%)$ & $R^{\sigma^{\text {raw }}}$ & $R^{\left\langle E^{\text {raw }}\right\rangle}(\%)$ & $R^{\sigma^{\text {raw }}}$ & $R^{\left\langle E^{\text {raw }}\right\rangle}(\%)$ & $R^{\sigma^{\text {raw }}}(\%)$ \\
\hline 1 & $\pi$ & 0.227 & 2.854 & 0.067 & 1.665 & 0.152 & 0.614 & 0.066 & 0.317 \\
\hline 2 & $\pi$ & 0.088 & 0.315 & 0.012 & 0.043 & 0.039 & 0.158 & 0.021 & 0.101 \\
\hline 3 & $\pi$ & 0.235 & 0.854 & 0.213 & 0.786 & 0.215 & 0.875 & 0.098 & 0.470 \\
\hline 4 & $\pi$ & 0.753 & 0.431 & 0.603 & 0.266 & 0.345 & 0.041 & 0.184 & 0.077 \\
\hline \multirow[t]{2}{*}{5} & $K$ & 0.221 & - & 0.029 & 0.145 & 0.005 & 0.011 & 0.047 & 0.049 \\
\hline & $p$ & - & 0.031 & 0.011 & 0.044 & 0.001 & 0.007 & - & 0.010 \\
\hline \multirow[t]{2}{*}{6} & $K$ & 0.002 & 0.006 & 0.067 & 0.377 & 0.152 & 0.682 & 0.038 & 0.207 \\
\hline & $p$ & 0.002 & 0.006 & 0.067 & 0.377 & 0.152 & 0.682 & 0.038 & 0.207 \\
\hline \multirow[t]{3}{*}{7} & $\pi$ & 1.138 & - & 1.138 & - & 1.138 & - & 1.138 & - \\
\hline & $K$ & 1.174 & - & 1.174 & - & 1.174 & - & 1.174 & - \\
\hline & $p$ & 1.234 & - & 1.234 & - & 1.234 & - & 1.234 & - \\
\hline
\end{tabular}

The effects of each of the seven considered sources of systematic uncertainties on the four energy determinations are correlated. In the study, correlation coefficient values equal to +1 were used.

The systematic uncertainties in Table 6 were obtained by combining in quadrature the effects of the seven sources reported in Table 7. Eleven values of the twelve energy response normalized to incident beam energy determinations have a total uncertainty smaller than $1.4 \%$. It is mainly defined by the uncertainty in the calibration of the energy response of the relatively small part of the calorimeter involved in the study. In the case of kaons with $E_{\text {beam }}=16$ $\mathrm{GeV}$, due to the large statistical uncertainty, the uncertainty on the determination of $R^{\left\langle E^{\text {raw }}\right\rangle}$, is equal to $2.4 \%$. Nine of the twelve determinations of the energy resolution normalized to incident beam energy, $R^{\sigma^{\text {raw }}}$, have a total uncertainty smaller than $1.9 \%$. The uncertainty values of the determinations of $R^{\sigma^{\text {raw }}}$ obtained in the case of $16 \mathrm{GeV}$ pion and kaon and $18 \mathrm{GeV}$ kaon beams are equal to $3.1 \%, 20.3 \%$ and $10.4 \%$, respectively.

The determinations of $R^{\left\langle E^{\text {raw }}\right\rangle}$ as a function of $E_{\text {beam }}$ and of $R^{\sigma^{\text {raw }}}$ as a function of $1 / \sqrt{E_{\text {beam }}[\mathrm{GeV}]}$ are reported in Figs. 20 and 21, respectively. In the case of experimental results, statistical and systematic uncertainties are combined in quadrature. In the case of simulated results, only statistical uncertainties are shown.

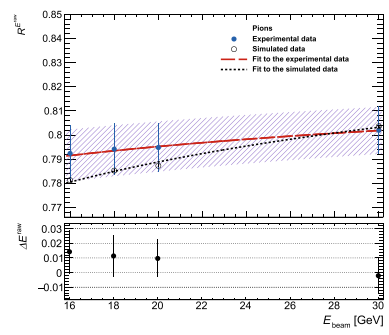

(a)

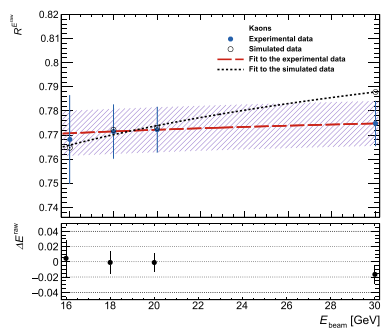

(b)

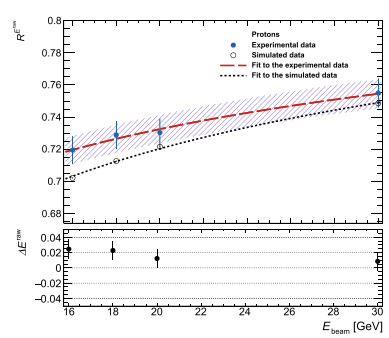

(c)

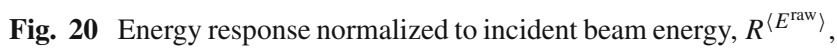
measured (blue circles) and predicted by MC simulation (black circles) as a function of beam energy obtained in the case of pion (a), kaon (b) and proton (c) beams. The experimental uncertainties include statistical and systematic effects combined in quadrature. Only statistical uncertainties affect simulated results. The red dashed (black dotted) curves are fits of the Eq. (22) to the experimental (simulated) data points. In case of experimental determinations the dashed blue hashed bands were obtained by using the correlated systematic uncertainties. In the bottom of the histograms are shown the fractional differences $\Delta E^{\langle\mathrm{raw}\rangle}$ defined in Eq. (12). The uncertainties include statistical and systematic effects combined in quadrature 


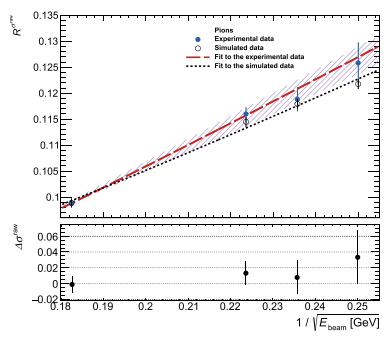

(a)

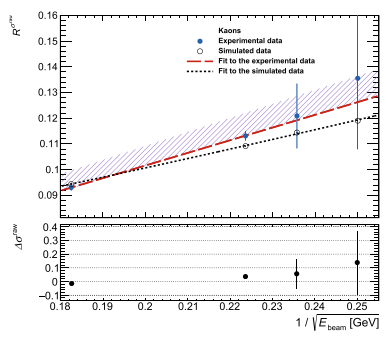

(b)

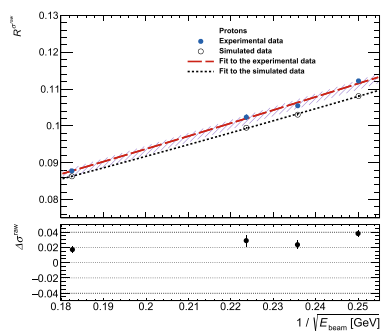

(c)

Fig. 21 Energy resolution normalized to incident beam energy, $R^{\sigma^{\text {raw }}}$, measured (blue circles) and predicted by MC simulation (black circles) as a function of $1 / \sqrt{E_{\text {beam }}}$ obtained in the case of pion (a), kaon (b) and proton (c) beams. The experimental uncertainties include statistical and systematic effects combined in quadrature. Only statistical uncertainties affect simulated results. The red dashed (black dotted) curves are fits of the Eq. (30) to the experimental (simulated) data points. In case of experimental determinations the dashed blue hashed bands were obtained by using the correlated systematic uncertainties. In the bottom of the histograms are shown the fractional differences $\Delta \sigma^{\text {raw }}$ defined in Eq. (13). The uncertainty includes statistical and systematic effects combined in quadrature

\subsection{Comparison between experimental and simulated results}

A quantitative comparison between experimental and simulated results can be obtained using the quantities

$\Delta\left\langle E^{\mathrm{raw}}\right\rangle=\frac{\left\langle E^{\mathrm{raw}}\right\rangle}{\left\langle E_{\mathrm{MC}}^{\mathrm{raw}}\right\rangle}-1$

and

$\Delta \sigma^{\text {raw }}=\frac{\sigma^{\text {raw }}}{\sigma_{\text {MC }}^{\text {raw }}}-1$

The results are reported in Table 8 where statistical and systematic uncertainties are shown separately. The statistical uncertainties include the experimental and simulated uncertainties combined in quadrature. The results are also shown in Figs. 20 and 21 where statistical and systematic uncertainties are combined in quadrature.

The average of the absolute values of the difference of all the energy response (resolution) measurements obtained using experimental and simulated data is $1.1 \%(3.4 \%)$. In the case of the response determinations and the resolution
Table 8 Comparison of the energy response (left) and resolution (right) obtained analyzing experimental and simulated data in the case of pion, kaon and proton beams with different beam energies. Statistical uncertainties (first value) and systematic uncertainties (second value) are reported.

\begin{tabular}{cll}
\hline $\begin{array}{c}E_{\text {beam }} \\
(\mathrm{GeV})\end{array}$ & $\Delta E^{\text {raw }}=\frac{\left\langle E^{\text {raw }}\right\rangle}{\left\langle E_{\mathrm{MC}}^{\text {raw }}\right\rangle}-1$ & $\Delta \sigma^{\text {raw }}=\frac{\sigma^{\text {raw }}}{\sigma_{\mathrm{MC}}^{\text {raw }}}-1$ \\
\hline \multicolumn{3}{c}{ Pions } \\
16 & $0.0142 \pm 0.0009 \pm 0.0146$ & $0.0333 \pm 0.0110 \pm 0.0317$ \\
18 & $0.0113 \pm 0.0008 \pm 0.0137$ & $0.0077 \pm 0.0102 \pm 0.0187$ \\
20 & $0.0097 \pm 0.0007 \pm 0.0128$ & $0.0131 \pm 0.0088 \pm 0.0113$ \\
30 & $-0.0021 \pm 0.0006 \pm 0.0121$ & $-0.0012 \pm 0.0090 \pm 0.0061$ \\
\multicolumn{3}{c}{ Kaons } \\
16 & $0.0045 \pm 0.0207 \pm 0.0122$ & $0.1385 \pm 0.2319 \pm 0.0001$ \\
18 & $-0.0009 \pm 0.0082 \pm 0.0120$ & $0.0565 \pm 0.1099 \pm 0.0042$ \\
20 & $-0.0010 \pm 0.0022 \pm 0.0121$ & $0.0363 \pm 0.0157 \pm 0.0071$ \\
30 & $-0.0166 \pm 0.0015 \pm 0.0118$ & $-0.0144 \pm 0.0125 \pm 0.0021$ \\
& Protons \\
16 & $0.0246 \pm 0.0008 \pm 0.0122$ & $0.0382 \pm 0.0048 \pm 0.0003$ \\
18 & $0.0228 \pm 0.0006 \pm 0.0123$ & $0.0235 \pm 0.0041 \pm 0.0039$ \\
20 & $0.0123 \pm 0.0005 \pm 0.0122$ & $0.0289 \pm 0.0037 \pm 0.0070$ \\
30 & $0.0085 \pm 0.0005 \pm 0.0121$ & $0.0171 \pm 0.0041 \pm 0.0021$ \\
\hline
\end{tabular}

determinations of pions and kaons, the differences are consistent within the uncertainties. The uncertainties of the proton resolution determinations are about one order of magnitude smaller.

\subsection{Comparison between pion, kaon and proton energy} responses and resolutions

The values of the ratios

$$
\begin{aligned}
& \frac{R^{\left\langle E^{\text {raw }}\right\rangle(K)}}{R^{\left\langle E^{\text {raw }}\right\rangle(\pi)}} \\
& \frac{R^{\sigma^{\text {raw }}(K)}}{R^{\sigma^{\text {raw }}(\pi)}} \\
& \frac{R^{\left\langle E^{\text {raw }}\right\rangle(p)}}{R^{\left\langle E^{\text {raw }}\right\rangle(\pi)}} \\
& \frac{R^{\sigma^{\text {raw }}(p)}}{R^{\text {raw }}(\pi)}
\end{aligned}
$$

obtained using experimental and simulated data are reported in Table 9. In the case of experimental results statistical and the systematic uncertainties are shown separately. The systematic uncertainty was obtained by combining in quadrature the contribution of the seven sources of systematic uncertainties discussed in Sect. 5.1. The uncertainty on the scale of the reconstructed cell energy, $C_{\mathrm{c}}^{\mathrm{EM}}$, affects in a correlated way the reconstruction of the energy deposited in the modules by pions, kaons and protons. It follow that its effects on the energy response ratio determinations cancel to a negligible 
Table 9 Values of the ratios (14)-(17) obtained using experimental and simulated data produced by particles with $E_{\text {beam }}$ equal to $16,18,20$ and $30 \mathrm{GeV}$. For experimental determinations statistical (first value) and correlated systematic uncertainties (second value) are reported separately. Only statistical uncertainties are shown for the MC results

\begin{tabular}{lcc}
\hline$E_{\text {beam }}[\mathrm{GeV}]$ & Experimental data & Simulation data \\
\hline & $R^{\left\langle E^{\text {raw }}\right\rangle}(K) / R^{\left\langle E^{\text {raw }}\right\rangle}(\pi)$ \\
16 & $0.9694 \pm 0.0199 \pm 0.0081$ & $0.9788 \pm 0.0007$ \\
18 & $0.9714 \pm 0.0080 \pm 0.0062$ & $0.9833 \pm 0.0007$ \\
20 & $0.9715 \pm 0.0021 \pm 0.0042$ & $0.9819 \pm 0.0007$ \\
30 & $0.9661 \pm 0.0014 \pm 0.0021$ & $0.9803 \pm 0.0006$ \\
& $R^{\left\langle E^{\text {raw }}\right\rangle}(p) / R^{\left\langle E^{\text {raw }}\right\rangle}(\pi)$ \\
16 & $0.9079 \pm 0.0008 \pm 0.0073$ & $0.8987 \pm 0.0007$ \\
18 & $0.9177 \pm 0.0006 \pm 0.0059$ & $0.9075 \pm 0.0006$ \\
20 & $0.9188 \pm 0.0004 \pm 0.0040$ & $0.9164 \pm 0.0006$ \\
30 & $0.9414 \pm 0.0004 \pm 0.0020$ & $0.9314 \pm 0.0005$ \\
& $R^{\sigma^{\text {raw }}}(K) / R^{\sigma^{\text {raw }}}(\pi)$ & \\
16 & $1.0769 \pm 0.2195 \pm 0.0331$ & $0.9774 \pm 0.0080$ \\
18 & $1.0172 \pm 0.1059 \pm 0.0193$ & $0.9702 \pm 0.0084$ \\
20 & $0.9752 \pm 0.0147 \pm 0.0127$ & $0.9533 \pm 0.0084$ \\
30 & $0.9418 \pm 0.0118 \pm 0.0061$ & $0.9544 \pm 0.0088$ \\
& $R^{\sigma^{\text {raw }}}(p) / R^{\sigma^{\text {raw }}}(\pi)$ & \\
16 & $0.8918 \pm 0.0074 \pm 0.0274$ & $0.8876 \pm 0.0072$ \\
18 & $0.8876 \pm 0.0059 \pm 0.0168$ & $0.8738 \pm 0.0075$ \\
20 & $0.8824 \pm 0.0029 \pm 0.0115$ & $0.8689 \pm 0.0076$ \\
30 & $0.8887 \pm 0.0033 \pm 0.0057$ & $0.8727 \pm 0.0079$ \\
\hline & & \\
& & \\
& &
\end{tabular}

level. In the case of simulated data only statistical uncertainties are reported. The determinations are also shown as a function of $E_{\text {beam }}$ in Fig. 22. The experimental data error bars were obtained combining in quadrature statistical and systematic uncertainties. In the case of simulated data the error bars correspond to statistical uncertainties.

In the considered $E_{\text {beam }}$ range, the measured ratios of the kaon over pion energy responses is constant with a weighted average equal to $0.967 \pm 0.002(-0.014)$. The value in parentheses indicates the difference between the experimental and the simulated results. The ratios of the energy responses of protons and pions range between $0.908 \pm 0.008$ $(+0.009)$ at $E_{\text {beam }}=16 \mathrm{GeV}$ to $0.941 \pm 0.001(+0.010)$ at $E_{\text {beam }}=30 \mathrm{GeV}$. The values of the ratios of the energy resolution determinations are constants. The weighted averages values are $R^{\sigma^{\text {raw }}}(K) / R^{\sigma^{\text {raw }}}(\pi)=0.95 \pm 0.01(-0.011)$ and $R^{\sigma^{\text {raw }}}(p) / R^{\sigma^{\text {raw }}}(\pi)=0.888 \pm 0.005(+0.011)$, respectively.

The results presented in this paper extend the energy range of the determinations of the ratio of the responses and resolutions of protons and pions down to $16 \mathrm{GeV}$; previous results obtained by ATLAS Collaboration considered beams above $50 \mathrm{GeV}$ [12]. In the momentum range from 3 to $300 \mathrm{GeV} / \mathrm{c}$, the energy response for protons was systematically lower

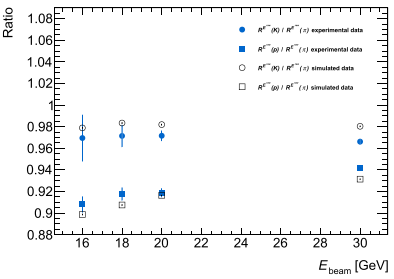

(a)

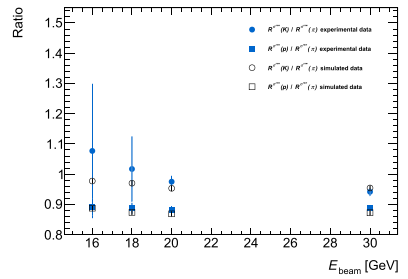

(b)
Fig. 22 a Ratios of the kaon and proton responses over those from the pions as a function of $E_{\text {beam }}$. The blue circles (black empty circles) show the ratios $R^{\left\langle E^{\text {raw }}\right\rangle}(K) / R^{\left\langle E^{\text {raw }}\right\rangle}(\pi)$ obtained using experimental (simulated) data. The blue full (black empty) squares show the ratios $R^{\left\langle E^{\text {raw }}\right\rangle}(p) / R^{\left\langle E^{\text {raw }}\right\rangle}(\pi)$ obtained using experimental (simulated) data. b Ratios of the kaon and proton resolutions over those from the pion as a function of $E_{\text {beam. }}$. The blue cicles (black empty circles) show the ratios $R^{\sigma^{\text {raw }}}(K) / R^{\sigma^{\text {raw }}}(\pi)$ obtained using experimental (simulated) data. The blue full (black empty) squares show the ratios $R^{\sigma^{\text {raw }}}(p) / R^{\sigma^{\text {raw }}}(\pi)$ obtained using experimental (simulated) data. In the case of experimental results uncertainties include statistical and systematic effects combined in quadrature. For simulated results, only statistical uncertainties are reported

than the $\pi^{ \pm}$one in the CMS result [15]. In the same paper results concerning the response of charged kaons and antiprotons for momenta below $9 \mathrm{GeV} / \mathrm{c}$ are reported.

\section{Comparison with hadronic cascade model predictions}

6.1 Parametrization of the energy response normalized to incident beam energy as a function of $E_{\text {beam }}$

The calorimeter energy response and resolution to pions, kaons and protons can be described in terms of the detector non-compensation and leading particle effects [16]. The hadron energy response normalized to incident beam energy as a function of the beam energy can be parametrized according to

$R^{\left\langle E^{\mathrm{raw}}\right\rangle}=\left(1-F_{\mathrm{h}}\right)+F_{\mathrm{h}} \times\left(\frac{e}{h}\right)^{-1}$

where $F_{\mathrm{h}}$ represents the non-electromagnetic energy component of showers and $e / h$ is the ratio between the responses to the purely EM and hadronic components of showers. The measurements allow a determination of the ratios of the nonelectromagnetic energy component of showers induced by incident pions $\left(F_{\mathrm{h}}(\pi)\right)$, kaons $\left(F_{\mathrm{h}}(K)\right)$ and protons $\left(F_{\mathrm{h}}(p)\right)$ for the same value of $E_{\text {beam. }}$. Using Eq. (18) one obtains

$\frac{F_{\mathrm{h}}(K)}{F_{\mathrm{h}}(\pi)}=\frac{1-R^{\left\langle E^{\mathrm{raw}}\right\rangle}(K)}{1-R^{\left\langle E^{\mathrm{raw}}\right\rangle}(\pi)}$

and

$\frac{F_{\mathrm{h}}(p)}{F_{\mathrm{h}}(\pi)}=\frac{1-R^{\left\langle E^{\mathrm{raw}}\right\rangle}(p)}{1-R^{\left\langle E^{\mathrm{raw}}\right\rangle}(\pi)}$ 
Table 10 Values of the ratios $F_{\mathrm{h}}(K) / F_{\mathrm{h}}(\pi)$ and $F_{\mathrm{h}}(p) / F_{\mathrm{h}}(\pi)$ obtained using experimental and simulated data for the four values of the beam energies $E_{\text {beam. }}$. In the case of experimental determinations statistical (first value) and systematic (second value) uncertainties are reported separately. Only statistical uncertainties affect the MC results

\begin{tabular}{ccc}
\hline$E_{\text {beam }}[\mathrm{GeV}]$ & Experimental Data & Simulated Data \\
\hline & $F_{\mathrm{h}}(K) / F_{\mathrm{h}}(\pi)$ \\
16 & $1.1165 \pm 0.0761 \pm 0.0354$ & $1.0756 \pm 0.0028$ \\
18 & $1.1102 \pm 0.0309 \pm 0.0276$ & $1.0608 \pm 0.0028$ \\
20 & $1.1101 \pm 0.0083 \pm 0.0186$ & $1.0669 \pm 0.0028$ \\
30 & $1.1371 \pm 0.0059 \pm 0.0098$ & $1.0805 \pm 0.0027$ \\
& $F_{\mathrm{h}}(p) / F_{\mathrm{h}}(\pi)$ \\
16 & $1.3512 \pm 0.0039 \pm 0.0418$ & $1.3617 \pm 0.0034$ \\
18 & $1.3173 \pm 0.0030 \pm 0.0326$ & $1.3382 \pm 0.0033$ \\
30 & $1.3144 \pm 0.0019 \pm 0.0217$ & $1.3091 \pm 0.0033$ \\
& $1.2373 \pm 0.0018 \pm 0.0107$ & $1.2807 \pm 0.0031$ \\
\hline
\end{tabular}

The experimental and simulated data results obtained using Eqs. (19) and (20) are reported in Table 10. The statistical and the systematic uncertainties are shown separately in the case of experimental results. The systematic uncertainties were derived by combining in quadrature the effects of the seven sources discussed in Sect. 5.1. For simulated data, only statistical uncertainties are reported. Experimental (simulated) data show constant ratios $F_{\mathrm{h}}(K) / F_{\mathrm{h}}(\pi)$ and the weighted average of these ratios is $1.13 \pm 0.01(1.072 \pm$ $0.001)$. The ratio $F_{\mathrm{h}}(p) / F_{\mathrm{h}}(\pi)$ decreases from $1.351 \pm 0.04$ $(1.361 \pm 0.003)$ at $E_{\text {beam }} 16 \mathrm{GeV}$ to $1.24 \pm 0.01(1.281 \pm$ $0.003)$ at $E_{\text {beam }} 30 \mathrm{GeV}$.

The ratio $F_{\mathrm{h}}(p) / F_{\mathrm{h}}(\pi)$, as obtained in Refs. [17,18] from the copper/quartz-fiber calorimeter data [19], varies from 1.22 at $200 \mathrm{GeV}$ to 1.15 at $370 \mathrm{GeV}$. In Ref. [20], a constant value of $F_{\mathrm{h}}(p) / F_{\mathrm{h}}(\pi)$ in the range between 1.15 and 1.20 is predicted.

The determinations of the ratios $F_{\mathrm{h}}(K) / F_{\mathrm{h}}(\pi)$ and $F_{\mathrm{h}}(p) / F_{\mathrm{h}}(\pi)$ as a function of $E_{\text {beam }}$ are also reported in Fig. 23a, b, respectively. In the case of experimental results, statistical and systematic uncertainties are combined in quadrature, but for simulated results only statistical uncertainty are shown.

Groom's parameterization $[17,18,20]$ predicts

$F_{\mathrm{h}}=\left(\frac{E_{\text {beam }}}{E_{0}}\right)^{m-1}$

where the quantity $E_{0}$ is the energy at which multiple pion production becomes significant and the parameter $m$ describes the relation between the average multiplicity of secondary particles produced in the collision and the fraction of energy going into $\pi^{0}$ s in one collision. Using Eq. (18) one obtains

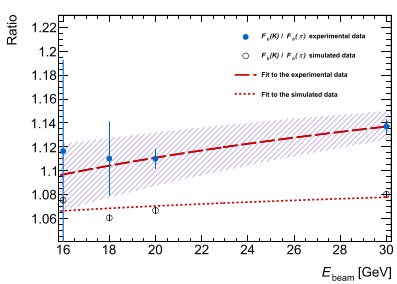

(a)

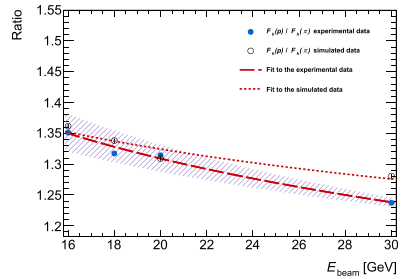

(b)
Fig. 23 a $F_{\mathrm{h}}(K) / F_{\mathrm{h}}(\pi)$ as a function of $E_{\text {beam }}$ obtained using experimental (blue circles) and simulated data (black empty circles). b $F_{\mathrm{h}}(p) / F_{\mathrm{h}}(\pi)$ as a function of $E_{\text {beam }}$ obtained using experimental (blue circles), and simulated data (black empty circles). In the case of experimental results uncertainties include statistical and systematic effects combined in quadrature, but for simulated results only the statistical uncertainties are shown. The dashed (dotted) red curves are fits of the functional forms (23) and (24) to the experimental (simulated) data points. In case of experimental determinations the dashed blue hashed bands were obtained by using the correlated systematic uncertainties

$R^{\left\langle E^{\text {raw }}\right\rangle}=1+\frac{1}{\left(E_{0}\right)^{m-1}}\left[\left(\frac{e}{h}\right)^{-1}-1\right]\left(E_{\text {beam }}\right)^{m-1}$.

and

$$
\begin{aligned}
\frac{F_{\mathrm{h}}(K)}{F_{\mathrm{h}}(\pi)} & =\frac{E_{0}(\pi)^{m(\pi)-1}}{E_{0}(K)^{m(K)-1}} \times\left(E_{\text {beam }}\right)^{m(K)-m(\pi)} \\
\frac{F_{\mathrm{h}}(p)}{F_{\mathrm{h}}(\pi)} & =\frac{E_{0}(\pi)^{m(\pi)-1}}{E_{0}(p)^{m(p)-1}} \times\left(E_{\text {beam }}\right)^{m(p)-m(\pi)}
\end{aligned}
$$

Fits of Eq. (23) to the histograms in Fig. 23a and of Eq. (24) to the histograms in Fig. 23b allow a determination of

$$
\begin{aligned}
B_{K / \pi} & =\frac{E_{0}(\pi)^{m(\pi)-1}}{E_{0}(K)^{m(K)-1}}, \\
C_{K / \pi} & =m(K)-m(\pi)
\end{aligned}
$$

and

$B_{p / \pi}=\frac{E_{0}(\pi)^{m(\pi)-1}}{E_{0}(p)^{m(p)-1}}$,

$C_{p / \pi}=m(p)-m(\pi)$,

respectively. The fit curves to the experimental and simulated data are show in the figure. The hashed bands were obtained using the correlated systematic uncertainties $\Delta F_{\text {syst. }}^{K}$ and $\Delta F_{\text {syst. }}^{p}$ reported in Table 10 on the determinations of the ratios (23) and (24), respectively. In Fig. 23a the band is defined by the curves fitting Eq. (23) to the points $F_{\mathrm{h}}(K) / F_{\mathrm{h}}(\pi)+\Delta F_{\text {syst. }}^{K}$ and $F_{\mathrm{h}}(K) / F_{\mathrm{h}}(\pi)-\Delta F_{\text {syst. }}^{K}$. The band in Fig. $23 \mathrm{~b}$ is defined by the curves obtained fitting Eq. (24) to the points $F_{\mathrm{h}}(p) / F_{\mathrm{h}}(\pi)+\Delta F_{\text {syst. }}^{p}$ and $F_{\mathrm{h}}(p) / F_{\mathrm{h}}(\pi)-\Delta F_{\text {syst. }}^{p}$. All the fits were performed using only statistical uncertainties. The values of the parameters obtained in the fits are reported in Table 11. The first uncertainty value is the statistical uncertainty corresponding to the square root of the diagonal term of the fit error matrix. The systematic uncertainty (second uncertainty value) is 
Table 11 Values of the parameters $B_{K / \pi}$ (Eq. (25)) and $C_{K / \pi}$ (Eq. (26)) obtained fitting Eq. (23) to the experimental and simulated values of $F_{\mathrm{h}}(K) / F_{\mathrm{h}}(\pi)$ as a function of $E_{\text {beam }}$ shown Fig. 23a. Values of the parameters $B_{p / \pi}$ (Eq. (27)) and $C_{p / \pi}$ (Eq. (28)) obtained fitting Eq. (24) to the experimental and simulated values of $F_{\mathrm{h}}(p) / F_{\mathrm{h}}(\pi)$ as a function of $E_{\text {beam }}$ shown Fig. 23b. In the case of simulated (experimental) data results, statistical (statistical and systematic) uncertainties are reported. The fit $\chi^{2}$ probability values are also shown

\begin{tabular}{lll}
\hline & Experimental data & Simulated data \\
\hline$B_{K / \pi}$ & $0.936 \pm 0.065 \pm 0.083$ & $1.014 \pm 0.004$ \\
$C_{K / \pi}$ & $0.057 \pm 0.021 \pm 0.023$ & $0.0182 \pm 0.0014$ \\
$\chi^{2}$ prob. & 0.944 & $1.767 \times 10^{-7}$ \\
\hline$B_{p / \pi}$ & $1.975 \pm 0.023 \pm 0.192$ & $1.735 \pm 0.0074$ \\
$C_{p / \pi}$ & $-0.137 \pm 0.004 \pm 0.027$ & $-0.090 \pm 0.0014$ \\
$\chi^{2}$ prob. & 0.311 & $8.17910 \times 10^{-7}$ \\
\hline
\end{tabular}

equal to half of the differences between the determinations obtained fitting Eq. (23) to the points $F_{\mathrm{h}}(K) / F_{\mathrm{h}}(\pi)+\Delta F_{\text {syst. }}^{K}$. and $F_{\mathrm{h}}(K) / F_{\mathrm{h}}(\pi)-\Delta F_{\text {syst. }}^{K}$ and Eq. (24) to the points $F_{\mathrm{h}}(p) / F_{\mathrm{h}}(\pi)+\Delta F_{\text {syst. }}^{p}$ and $F_{\mathrm{h}}(p) / F_{\mathrm{h}}(\pi)-\Delta F_{\text {syst. }}^{p}$, respectively. In the table, the fit $\chi^{2}$ probability values are reported. In the case of simulated data fits the probabilities are very small.

The values of $B_{K / \pi}$ and $C_{K / \pi}$ obtained using experimental and simulated data agree within two sigmas. The values of $B_{p / \pi}$ and $C_{p / \pi}$ obtained using experimental and simulated data differ significantly.

Fits of Eq. (22) to the determined values of $R^{\left\langle E^{\text {raw }}\right\rangle}$ as a function of $E_{\text {beam }}$ (Fig. 20) allows for the determination [17] of $m$ and

$A=\frac{1}{\left(E_{0}\right)^{m-1}}\left[\left(\frac{e}{h}\right)^{-1}-1\right]$.

The resulting fits to the experimental and simulated determinations are overlapped in Fig. 20. The hashed bands were obtained using the correlated systematic uncertainties $\Delta R_{\text {syst. }}^{E^{\text {raw }}}$ reported in Table 6 . They are bounded by the curves obtained fitting Eq. (22) to the points $R^{E^{\text {raw }}}+\Delta R_{\text {syst. }}^{E^{\text {raw }}}$ and $R^{E^{\text {raw }}}-\Delta R_{\text {syst. }}^{E^{\text {raw }}}$. All fits are performed accounting for the statistical uncertainties on the data points. The obtained values of $A$ and $m$ are reported in Table 12. The first uncertainty value is the statistical uncertainty. It corresponds to the square root of the diagonal term of the error matrix. The systematic uncertainty (second uncertainty value) is equal to half of the differences between the determinations obtained fitting Eq. (22) to the points $R^{E^{\text {raw }}}+\Delta R_{\text {syst. }}^{E_{\text {raw }}}$ and $R^{E^{\text {raw }}}-\Delta R_{\text {syst. }}^{E^{\text {raw }}}$. The fit $\chi^{2}$ probability values are reported. In the case of the fits to kaon and proton simulated data the probabilities are very small.
Table 12 Values of the parameters $A$ (Eq. (29)) and $m$ obtained fitting Eq. (22) to the experimental and simulated energy response normalized to incident beam energy, $R^{E^{\text {raw }}}$, as a function of $E_{\text {beam. The fit }}$ functions are overlapped to the determinations in Fig. 20. In the case of experimental data results statistical and systematic uncertainties are reported. Only statistical uncertainties appear in the case of simulated data results. The $\chi^{2}$ probability values of the fits are reported

\begin{tabular}{lll}
\hline & Experimental data & Simulated data \\
\hline Pions & & \\
$A$ & $-0.2612 \pm 0.0019 \pm 0.0165$ & $-0.3557 \pm 0.0052$ \\
$m$ & $0.9187 \pm 0.0023 \pm 0.0041$ & $0.8258 \pm 0.0048$ \\
$\chi^{2}$ prob. & 0.004 & 0.238 \\
Kaons & & \\
$A$ & $-0.2481 \pm 0.0171 \pm 0.0095$ & $-0.3620 \pm 0.0025$ \\
$m$ & $0.9715 \pm 0.0210 \pm 0.0008$ & $0.8428 \pm 0.0023$ \\
$\chi^{2}$ prob. & 0.981 & $2.634 \times 10^{-14}$ \\
Protons & & \\
$A$ & $-0.5041 \pm 0.0044 \pm 0.0005$ & $-0.6208 \pm 0.0031$ \\
$m$ & $0.7885 \pm 0.0029 \pm 0.0004$ & $0.7338 \pm 0.0016$ \\
$\chi^{2}$ prob. & 0.632 & $4.295 \times 10^{-12}$ \\
\hline
\end{tabular}

The values of $m$ obtained using pions, kaons and protons data without making any assumption on the values of $e / h$ and $E_{0}$ are: $0.919 \pm 0.005(0.826 \pm 0.005), 0.97 \pm$ $0.02(0.843 \pm 0.002)$ and $0.789 \pm 0.003(0.734 \pm 0.002)$, respectively. The values in parenthesis were obtained using simulated data. According to Ref. [17] values of $m$ around 0.87 are expected. The determinations can be compared with previous pion measurements summarized in [17].

To compare the results discussed in this paper with the ones obtained previously using pions beams with energy in the range $10-350 \mathrm{GeV}$ and incident in the TileCal modules at $\eta=0.35$ [12], Eq. (22) was fit to the pion determinations fixing $E_{0}=1 \mathrm{GeV}$. The obtained values $e / h=1.3535 \pm 0.0304$ and $m=0.9187 \pm 0.0047$ agree with the previous determination $1.33 \pm 0.02$ and $0.85 \pm 0.03$, respectively [12]. The uncertainties include statistical and systematic uncertainties combined in quadrature.

\subsection{Parametrization of the energy resolution as a function} of $E_{\text {beam }}$

The resolution of the energy measurements as a function of the beam energy $E_{\text {beam }}$ can be parametrized according to

$R^{\sigma^{\text {raw }}}=\frac{a}{\sqrt{E_{\text {beam }}}} \oplus b$

where $a / \sqrt{E_{\text {beam }}}$ describes the fluctuations on the number of particle produced in the showers, $b$ describes the nonuniformity of the cell response and the symbol $\oplus$ indicates 
Table 13 Values of the parameters $a$ and $b$ obtained fitting Eq. (30) to the experimental and simulated fractional resolution values $R^{\sigma^{\text {raw }}}$ obtained using pions $(\pi)$, kaons $(K)$ and protons $(p)$ as a function of $1 / \sqrt{E_{\text {beam }}[\mathrm{GeV}]}$ (Fig. 21). In the case of experimental data results, statistical and systematic uncertainties are reported. Only statistical uncertainties are shown for simulated data. The $\chi^{2}$ probability values of the fits are reported. Previous pion results are also shown $(\pi[12])$

\begin{tabular}{|c|c|c|c|}
\hline & $a\left(\% \mathrm{GeV}^{-1 / 2}\right)$ & $b(\%)$ & $\chi^{2}$ prob. \\
\hline \multicolumn{4}{|c|}{ Experimental data } \\
\hline$\pi$ & $46.68 \pm 0.30 \pm 2.22$ & $4.99 \pm 0.11 \pm 0.58$ & 0.941 \\
\hline$K$ & $49.9 \pm 2.60 \pm 2.46$ & $1.78 \pm 2.78 \pm 1.03$ & 0.935 \\
\hline$p$ & $40.28 \pm 0.38 \pm 0.08$ & $4.79 \pm 0.15 \pm 1.44$ & 0.007 \\
\hline$\pi[12]$ & $52.9 \pm 0.9$ & $5.7 \pm 0.2$ & - \\
\hline \multicolumn{4}{|c|}{ Simulated data } \\
\hline Pions & $42.25 \pm 1$ & $6.2 \pm 0.4$ & 0.0008 \\
\hline Kaons & $42.8 \pm 0.3$ & $5.3 \pm 0.1$ & 0.058 \\
\hline Protons & $38.05 \pm 0.23$ & $5.12 \pm 0.08$ & 0.392 \\
\hline
\end{tabular}

the sum in quadrature. In the considered beam energy range the noise contribution is negligible (Sect. 3.4).

The curves in Fig. 21 were obtained fitting Eq. (30) to the experimental and simulated determinations of $R^{\sigma^{\text {raw }}}$ as a function of $1 / \sqrt{E_{\text {beam }}[\mathrm{GeV}]}$. The hashed bands in the figure were obtained by using correlated systematic uncertainties $\Delta R_{\text {syst. }}^{\sigma^{\text {raw }}}$ reported in Table 6 . They are defined by the curves obtained fitting Eq. (30) to the points $R^{\sigma^{\text {raw }}}+\Delta R_{\text {syst. }}^{\sigma^{\text {raw }}}$ and $R^{\sigma^{\text {raw }}}-\Delta R_{\text {syst. }}^{\sigma^{\text {raw }}}$. All fits were performed using only statistical uncertainties. The resulting values of $a$ and $b$ are reported in Table 13. The statistical uncertainty is equal to the square root of the corresponding diagonal term of the fit error matrix. The systematic uncertainty is equal to half of the differences of the determinations obtained fitting Eq. (30) to the points $R^{\sigma^{\text {raw }}}+\Delta R_{\text {syst. }}^{\sigma^{\text {raw }}}$ and $R^{\sigma^{\text {raw }}}-\Delta R_{\text {syst. }}^{\sigma_{\text {raw }}}$. In the table the $\chi^{2}$ probability values of the fits performed to the central values are reported.

The values of $a$ obtained analyzing pion and kaon data are consistent within the large uncertainties of about $4 \%$. The value obtained using protons is $14 \%$ smaller. The constant term $b$ is about $5 \%$ and is consistent between the three particle beams. Similar extraction using simulated data results in $10 \%$ smaller values for $a$ while the values for $b$ are $30 \%$ larger.

The values of $a$ and $b$ obtained analyzing pion data are consistent within about 2.6 sigmas with the results obtained in Ref. [12].

\section{Summary and conclusions}

The results described in this paper were obtained by exposing three modules of the ATLAS Tile Calorimeter to positive pion and kaon and proton beams with energies equal to 16 ,
18, 20 and $30 \mathrm{GeV}$ and incident at the centre of the front face of a calorimeter module cell with an angle of $14^{\circ}$ from the normal. Two Cherenkov counters in the beam line made it possible to identify pions, kaons and protons. The effects of electrons contaminating the pion samples in reconstructing the pion energy were determined by exploiting the difference of electromagnetic and hadronic shower profiles in the detector.

The main purpose of this study is to compare the measured energy of particles with the predictions of the Geant4-based simulation program used in ATLAS to simulated jets produced in proton-proton collisions at the LHC.

Eleven (Nine) determinations of the twelve energy responses (resolutions) normalized to incident beam energy have a total uncertainty smaller than $1.4 \%$ (1.9\%). For kaons with $E_{\text {beam }}$ $=16 \mathrm{GeV}$, due to the large statistical error, the uncertainty on the determination of $R^{\left\langle E^{\text {raw }}\right\rangle}$, is equal to $2.4 \%$. The uncertainty values of the determinations of $R^{\left\langle\sigma^{\text {raw }}\right\rangle}$ obtained in the case of $16 \mathrm{GeV}$ pion and kaon and $18 \mathrm{GeV}$ kaon beams are equal to $3.1 \%, 20.3 \%$ and $10.4 \%$ respectively.

Determinations of all the energy responses and of the pion and kaon energy resolutions obtained using experimental and simulated data agree within the uncertainties. The average of the absolute values of the differences between the energy response measurements was found to be $1.1 \%$ with an average total uncertainty of $1.4 \%$. The average difference between all the resolution measurements was found to be $3.4 \%$. The average total uncertainty of proton (pion and kaon) resolution measurements is $0.6 \%(5.6 \%)$.

In the considered $E_{\text {beam }}$ range, the measured ratios of the kaon over pion energy responses is constant with a weighted average equal to $0.967 \pm 0.002(-0.014)$. In parenthesis the difference between the experimental and simulated results is shown. The ratio of the energy responses of protons and pions ranges between $0.908 \pm 0.008(+0.009)$ at $E_{\text {beam }}=16 \mathrm{GeV}$ to $0.941 \pm 0.001(+0.010)$ at $E_{\text {beam }}=30 \mathrm{GeV}$. The values of the ratios of the energy resolution determinations are constants. The weighted averages values are $R^{\sigma^{\text {raw }}}(K) / R^{\sigma^{\text {raw }}}(\pi)$ $=0.95 \pm 0.01(-0.011)$ and $R^{\sigma^{\text {raw }}}(p) / R^{\sigma^{\text {raw }}}(\pi)=0.888 \pm$ $0.005(+0.011)$.

The differences between pion, kaon and proton responses and resolutions result from the different fraction of nonelectromagnetic energy deposited by incident particles: $F_{\mathrm{h}}(\pi), F_{\mathrm{h}}(K)$ and $F_{\mathrm{h}}(p)$ and to the non-compensating nature of the detector. Experimental (simulated) data from the different beam energies have consistent $F_{\mathrm{h}}(K) / F_{\mathrm{h}}(\pi)$ and the resulting weighted average numerical is $1.13 \pm 0.01(1.072$ $\pm 0.001)$. The ratio $F_{\mathrm{h}}(p) / F_{\mathrm{h}}(\pi)$ decreases from $1.351 \pm$ $0.04(1.361 \pm 0.003)$ at $E_{\text {beam }} 16 \mathrm{GeV}$ to $1.24 \pm 0.01(1.281$ $\pm 0.003)$ at $E_{\text {beam }} 30 \mathrm{GeV}$.

The non-electromagnetic energy component of showers can be expressed in terms of the parameters $m$ and $E_{0}[\mathrm{GeV}]$. The ratio between the responses to purely EM and hadronic 
components of showers $e / h$ describes the non-compensation nature of the calorimeter (Sect. 6.1). The values of $m$ obtained using experimental (simulated) pions, kaons and protons data are $0.919 \pm 0.005(0.826 \pm 0.005), 0.97 \pm 0.02(0.843 \pm$ $0.002)$ and $0.789 \pm 0.003$ (0.734 \pm 0.002$)$, respectively.

As discussed in Sect. 6.2, the energy resolution as a function of the beam energy can be parametrized with a statistical term $a / \sqrt{E_{\text {beam }}[\mathrm{GeV}]}$ and a constant terms $b$. The values of $a\left[\% \mathrm{GeV}^{-1 / 2}\right]$ obtained analysing pions, kaons and protons are $47 \pm 2(42 \pm 1), 50 \pm 3(42.8 \pm 0.3)$ and $40.3 \pm 0.4$ (38.1 \pm 0.2$)$, respectively. The values in parenthesis were obtained analysing simulated data. The corresponding $b[\%]$ values are $5.0 \pm 0.6(6.2 \pm 0.4), 2 \pm 3(5.3 \pm 0.1)$ and 5 $\pm 1(5.12 \pm 0.08)$.

Data Availability Statement This manuscript has associated data in a data repository. [Authors'comment: Data included in this manuscript are available at https://www.hepdata.net/record/106116.]

Open Access This article is licensed under a Creative Commons Attribution 4.0 International License, which permits use, sharing, adaptation, distribution and reproduction in any medium or format, as long as you give appropriate credit to the original author(s) and the source, provide a link to the Creative Commons licence, and indicate if changes were made. The images or other third party material in this article are included in the article's Creative Commons licence, unless indicated otherwise in a credit line to the material. If material is not included in the article's Creative Commons licence and your intended use is not permitted by statutory regulation or exceeds the permitted use, you will need to obtain permission directly from the copyright holder. To view a copy of this licence, visit http://creativecomm ons.org/licenses/by/4.0/.

Funded by SCOAP ${ }^{3}$.

\section{References}

1. ATLAS Collaboration, JINST 3, S08003 (2008). https://doi.org/ 10.1088/1748-0221/3/08/S08003

2. CERN EN Engineering Department. H8 beam line (2020). http://sba.web.cern.ch/sba/BeamsAndAreas/resultbeam.asp? beamline $=\mathrm{H} 8$

3. S. Agostinelli et al., Nucl. Instrum. Methods A 506, 250 (2003). https://doi.org/10.1016/S0168-9002(03)01368-8

4. J. Allison et al., IEEE Trans. Nucl. Sci. 53, 270 (2006). https://doi. org/10.1109/TNS.2006.869826

5. D. Costanzo, A. Dell'Acqua, A. Di Simone, M. Gallas, A. Nairz, A. Rimoldi, J. Boudreau, V. TSulaia, ATLAS detector simulation: status and outlook. Tech. Rep. ATL-SOFT-PUB-2005-004. CERNATL-SOFT-PUB-2005-004. ATL-COM-SOFT-2005-008. CERN, Geneva (2005). https://cds.cern.ch/record/916030
6. J. Spanggaard, Delay Wire Chambers - A Users Guide. Tech. Rep. SL-Note-98-023-BI. CERN, Geneva (1998). http://cds.cern. $\mathrm{ch} / \mathrm{record} / 702443$

7. B. Di Girolamo, A. Dotti, V. Giangiobbe, P. Johansson, L. Pribyl, M. Volpi, Beamline instrumentation in the 2004 combined ATLAS testbeam. Tech. Rep. ATL-TECH-PUB-2005-001. ATLCOM-TECH-2005-001. CERN, Geneva (2005). http://cds.cern.ch/ record/831497

8. ATLAS Collaboration, Technical Design Report for the PhaseII Upgrade of the ATLAS Tile Calorimeter. Tech. Rep. CERNLHCC-2017-019. ATLAS-TDR-028. CERN, Geneva (2017). http://cds.cern.ch/record/2285583

9. M. Crouau, P. Grenier, G. Montarou, S. Poirot, F. Vazeille, Characterization of 8-stages Hamamatsu R5900 photomultipliers for the TILE calorimeter. Tech. Rep. ATL-TILECAL-97-129. ATL-L-PN129. CERN, Geneva (1997). http://cds.cern.ch/record/683595

10. J. Abdallah, et al., JINST 8, 26 (2007) (P01005)

11. A. Valero, The ATLAS TileCal read-out drivers signal reconstruction. Tech. Rep. ATL-TILECAL-PROC-2009-004. CERN, Geneva (2009). http://cds.cern.ch/record/1223960

12. P. Adragna et al., Nucl. Instrum. Methods A606, 362 (2009). https:// doi.org/10.1016/j.nima.2009.04.009

13. ATLAS Collaboration, Eur. Phys. J. C 78(12), 987 (2018). https:// doi.org/10.1140/epjc/s10052-018-6374-z

14. H.W. Bertini, M.P. Guthrie, Nucl. Phys. A 169, 670 (1971). https:// doi.org/10.1016/0375-9474(71)90710-X

15. S. Abdullin, et al., Eur. Phys. J. C 60, 359 (2009). https:// doi.org/10.1140/epjc/s10052-009-1024-0 [Erratum: Eur. Phys. J.C61,353(2009)]

16. R. Wigmans, Int. Ser. Monogr. Phys. 107, $1(2000)$

17. D.E. Groom, Nucl. Instrum. Methods A 572(2), 633 (2007). https:// doi.org/10.1016/j.nima.2006.11.070. http://www.sciencedirect. com/science/article/pii/S0168900206023965

18. D.E. Groom, Nucl. Instrum. Methods A 593(3), 638 (2008). https:// doi.org/10.1016/j.nima.2008.05.045. http://www.sciencedirect. com/science/article/pii/S0168900208007924

19. N. Akchurin, S. Ayan, G. Bencze, K. Chikin, H. Cohn, S. Doulas, I. Dumanoğlu, E. Eskut, A. Fenyvesi, A. Ferrando, M. Fouz, O. Ganel, V. Gavrilov, Y. Gershtein, C. Hajdu, J. Iosifidis, M. Josa, A. Kayis, A. Khan, S. Kim, V. Kolosov, S. Kuleshov, A. Kuzucu-Polatoz, J. Langland, D. Litvintsev, J.P. Merlo, J. Molnar, A. Nikitin, Y. Onel, G. Önengüt, D. Osborne, N. Özdeş Koca, H. Oztürk, A. Penzo, E. Pesen, V. Podrasky, A. Rosowsky, J. Salicio, C. Sanzeni, R. Sever, H. Silvestri, V. Stolin, L. Sulak, J. Sullivan, A. Ulyanov, S. Uzunian, G. Vesztergombi, R. Wigmans, D. Winn, R. Winsor, A. Yumashev, P. Zalan, M. Zeyrek, Nucl. Instrum. Methods A 408(2), 380 (1998). https://doi.org/10.1016/ S0168-9002(98)00021-7. http://www.sciencedirect.com/science/ article/pii/S0168900298000217

20. T. Gabriel, D. Groom, P. Job, N. Mokhov, G. Stevenson, Nucl. Instrum. Methods A 338(2), 336 (1994). https://doi.org/ 10.1016/0168-9002(94)91317-X. http://www.sciencedirect.com/ science/article/pii/016890029491317X 